\title{
Structural Basis for the Calmodulin-Mediated Activation of eEF-2K
}

Andrea Piserchio ${ }^{1}$, Eta A. Isiorho ${ }^{2}$, Kimberly Long ${ }^{3}$, Amanda L. Bohanon ${ }^{4}$, Eric A. Kumar ${ }^{5}$,

Nathan Will ${ }^{1,6,9}$, David Jeruzalmi ${ }^{1,6}$, Kevin N. Dalby ${ }^{3,4,5 *}$ and Ranajeet Ghose ${ }^{1,6,7,8 *}$

${ }^{1}$ Department of Chemistry and Biochemistry, The City College of New York, New York, NY 10031.

${ }^{2}$ Macromolecular Crystallization Facility, CUNY ASRC, New York, NY 10031.

${ }^{3}$ Graduate Program in Cell and Molecular Biology, University of Texas, Austin, TX 78712.

${ }^{4}$ Graduate Program in Biochemistry, University of Texas, Austin, TX 78712.

${ }^{5}$ Division of Chemical Biology and Medicinal Chemistry, University of Texas, Austin, TX 78712.

${ }^{6} \mathrm{PhD}$ Program in Biochemistry, The Graduate Center of CUNY, New York, NY 10016.

${ }^{7}$ PhD Program in Chemistry, The Graduate Center of CUNY, New York, NY 10016.

${ }^{8} \mathrm{PhD}$ Program in Physics, The Graduate Center of CUNY, New York, NY 10016.

*Correspondence: dalby@austin.utexas.edu and rghose@ccny.cuny.edu

${ }^{\top}$ Current Address: Laboratory of Molecular Electron Microscopy, the Rockefeller University, New York, NY 10065. 


\begin{abstract}
Translation is a highly energy consumptive process ${ }^{1}$ tightly regulated for optimal protein quality $^{2}$ and adaptation to energy and nutrient availability. A key facilitator of this process is the $\alpha$-kinase eEF-2K that specifically phosphorylates the GTP-dependent translocase eEF-2, thereby reducing its affinity for the ribosome and suppressing the elongation phase of protein synthesis ${ }^{3,4}$. eEF-2K activation requires calmodulin binding and auto-phosphorylation at the primary stimulatory site, T348. Biochemical studies have predicted that calmodulin activates eEF-2K through a unique allosteric process $^{5}$ mechanistically distinct from other calmodulin-dependent kinases $^{6}$. Here we resolve the atomic details of this mechanism through a $2.3 \AA$ crystal structure of the heterodimeric complex of calmodulin with the functional core of eEF-2K (eEF-2 $\left.\mathrm{K}_{\mathrm{TR}}\right)$. This structure, which represents the activated T348-phosphorylated state of eEF-2K $\mathrm{K}_{\mathrm{TR}}$, highlights how through an intimate association with the calmodulin C-lobe, the kinase creates a "spine" that extends from its N-terminal calmodulin-targeting motif through a conserved regulatory element to its active site. Modification of key spine residues has deleterious functional consequences.
\end{abstract}




\section{Introduction}

Eukaryotic protein levels are predominantly controlled by mRNA translation ${ }^{7}$, an energetically expensive process ${ }^{1}$ that is highly regulated ${ }^{8}$ to ensure optimal transit times and maintain protein quality ${ }^{2}$, and fashion the cellular response to environmental stress and changes in energy/nutrient availability ${ }^{9}$. A primary driver of this regulation is the specific phosphorylation (on Thr-56) of the GTP-dependent translocase, eukaryotic elongation factor 2 (eEF-2). This covalent modification, uniquely catalyzed by the $\alpha$-kinase ${ }^{10}$, eukaryotic elongation factor 2 kinase $(\mathrm{eEF}-2 \mathrm{~K})^{11,12}$, diminishes the ability of eEF-2 to engage the ribosome suppressing the elongation phase of protein synthesis ${ }^{3,4}$. Dysregulation of eEF-2K activity has been linked to neurological conditions such as Alzheimer's-related dementia ${ }^{13}$ and Parkinson's disease ${ }^{14}$. Aberrant eEF-2K function has been correlated to enhanced tumorigenesis ${ }^{15}$, invasion, and metastasis ${ }^{16}$. eEF- $2 \mathrm{~K}$ is, therefore, an emerging target for the development of therapeutics against several diseases, including many cancers ${ }^{17}$, and neuropathies ${ }^{18}$, underscoring the importance of understanding its regulation in mechanistic detail.

eEF-2K is the only $\alpha$-kinase reliant on calmodulin $(\mathrm{CaM})^{11}$ for activation through an allosteric process ${ }^{5}$. CaM binding enhances the apparent $k_{\text {cat }}$ towards a peptide-substrate by $\sim 2400$ fold without significantly altering affinity. Subsequent auto-phosphorylation at the primary upregulating site (T348) provides an additional $\sim 6$-fold increase in $k_{\text {cat }}$, yielding the fully activated state. This mechanism is distinct from other CaM-dependent kinases that rely on CaM-induced displacement of an auto-inhibitory segment for enhanced substrate access ${ }^{6}$. eEF-2K activity is further modulated by $\mathrm{Ca}^{2+19}, \mathrm{pH}^{20}$, and regulatory phosphorylation ${ }^{21}$ via multiple pathways, including mTOR, AMPK, and ERK ${ }^{15}$. Obtaining insight into eEF-2K activation and regulation in atomic detail has been hindered by the absence of structures of the full-length enzyme or its CaM- 
bound activated complex. We have identified a minimal functional eEF-2K construct, eEF-2 $\mathrm{K}_{\mathrm{TR}}$

(Fig. 1a, Extended Data Fig. 1), activated by CaM similarly to the wild-type enzyme and efficiently phosphorylates eEF-2 in cells ${ }^{22}$. Using X-ray crystallography, we resolved the structure of its T348-phosphorylated state $\left(p \mathrm{eEF}-2 \mathrm{~K}_{\mathrm{TR}}\right)$ in an activated heterodimeric complex with CaM $\left(\mathrm{CaM} \cdot p \mathrm{eEF}-2 \mathrm{~K}_{\mathrm{TR}}\right)$ to $2.3 \AA$. Almost four decades after its discovery and characterization ${ }^{11}$, this structure provides the first critical insight into the atomic details of the unique mechanism of the CaM-mediated activation of eEF-2K.

\section{Results and discussion}

\section{Structural features of $p \mathrm{eF}-2 \mathrm{~K}_{\mathrm{TR}}$}

The CaM•peEF-2K $\mathrm{K}_{\mathrm{TR}}$ complex forms an elongated structure (Fig. 1b) consistent with previous solution-state X-ray scattering (SAXS) measurements on the inactive complex (unphosphorylated T348) 22 (Extended Data Fig. 2). The kinase domain (KD) shows a characteristic $\alpha$-kinase architecture ${ }^{23,24}$ with the "catalytic" residues in orientations suggestive of an active conformation (Extended Data Fig. 3a). While the P- and N/D-loops are closed (Extended Data Fig. 3b), as in the nucleotide-bound states of the related MHCK-A KD ${ }^{24,25}$, no density corresponding to nucleotide was apparent despite crystallization with the ATP-analog, AMP-PNP. Phosphorylated T348 ( $p$ T348; Extended Data Fig. 4) docks into a phosphate-binding pocket (PBP) hydrogen-bonding with the K205, R252, and T254 side-chains, and the L253 and T254 mainchains (Fig. 2a). D280 on the catalytic-loop hydrogen bonds with the side-chains of R252 and Y282. This interaction promotes methyl- $\pi$ interactions between I275 and Y282 (Fig. 2a), thereby coupling the catalytic site to the PBP.

The all-helical C-terminal domain (CTD) represents a feature unique to eEF-2K that forms a docking platform for eEF-2 26,27 . The $\alpha 2^{\prime}$ - $\alpha 7^{\prime}$ 'region displays a pattern resembling SEL1-like 
repeats $^{28}$, the $\alpha 8^{\prime}-\alpha 10^{\prime}$ 'segment deviates from this arrangement, and $\alpha 1^{\prime}$ is somewhat disengaged from the rest of the CTD. $\alpha 2$ ' stabilizes the KD/CTD interface, interacting with $\alpha \mathrm{E}$ on the KD Clobe $\left(\mathrm{KD}_{\mathrm{C}}\right)$ and $\alpha 3^{\prime}$ (Fig. $\left.2 \mathrm{~b}\right)$. The $\alpha \mathrm{E}-\alpha 2^{\prime}$ interface features hydrophobic interactions involving Y533 sandwiched by A306 and L307, and V526 inserted between M305 and L523. A curious feature within the $\alpha E-\alpha 2^{\prime}-\alpha 3^{\prime}$ element is the linear spatial positioning of three conserved His residues (H527 and H534 on $\alpha 2$ '; H554 on $\alpha 3$ '; Fig. 2b); the also spatially proximal H557 stacks with H527. H554 forms a rare $\delta$ - $\delta$ hydrogen bond with $\mathrm{H} 527^{29}$. H534 and H554 form salt bridges with the conserved E332 on a helical R-loop segment ( $\alpha$ L'). It is tempting to speculate that alternative protonation states of one or more of these His residues would modify interactions within the KD/CTD interface, and perhaps its coupling to the R-loop, through the $\alpha E-\alpha 2^{\prime}-\alpha 3^{\text {' }}$ element, and thereby contribute to the observed $\mathrm{pH}$ response of the enzyme ${ }^{20}$. NMR studies ${ }^{27}$ have localized the eEF-2 docking-site to the C-terminus of the CTD ( $\left.\alpha 9^{\prime}-\alpha 10^{\prime}\right), \sim 60 \AA$ away from the active site, suggesting that distinct interactions drive eEF-2 recognition and its subsequent phosphorylation.

\section{Recognition of peEF-2K $K_{\mathrm{TR}}$ by CaM}

CaM contacts peEF- $2 \mathrm{~K}_{\mathrm{TR}}$ in an extended conformation using both its $\mathrm{N}-\left(\mathrm{CaM}_{\mathrm{N}}\right)$ and $\mathrm{C}$ terminal $\left(\mathrm{CaM}_{\mathrm{C}}\right)$ lobes, with $\mathrm{CaM}_{\mathrm{C}}$ being more intimately associated with the kinase (Fig. 3a). This assembly is consistent with changes in protection seen in previous hydrogen exchange mass spectrometry (HXMS) analyses (Extended Data Fig. 5) 22,30 . All four $\mathrm{CaM}_{\mathrm{C}}$ helices engage the CaM-targeting motif (CTM) through their hydrophobic faces in a $\mathrm{Ca}^{2+}$-bound "open" conformation, and all, except $\alpha_{H}$, interact with the $\mathrm{N}$-lobe of the $\mathrm{KD}\left(\mathrm{KD}_{\mathrm{N}}\right)$ using their predominantly hydrophilic faces. A deep hydrophobic pocket, lined by the side-chains of Ala88 (three-letter codes used for CaM residues), Phe92, Ile100, Leu105, Met124, Ile125, Val136, 
Phe141, Met144, and Met145, accommodates W85 from the eEF-2K $\mathrm{KR}_{\mathrm{T}}$ CTM. Additional hydrophobic interactions of I89 with Ala88 and Met145, and of A92 with Val91 and Leu112 generate a 1-5-8 binding mode (Fig. 3b) within a helical CTM ( $\alpha$ CTM) like in the NMR structure of the complex of CaM with a peptide encoding the CTM (CTM-pep, 74-100) ${ }^{31}$. However, no $\mathrm{CaM}_{\mathrm{C}}$-bound $\mathrm{Ca}^{2+}$ ions were seen in the peptide complex ${ }^{31}$. The hydrophilic face of $\mathrm{CaM}_{\mathrm{C}}$ makes numerous contacts with $\mathrm{KD}_{\mathrm{N}}$ over a large interaction surface (Fig. 3c). A "virtual alanine scan" at the interface identified several contacts involving residues for which in silico Ala mutations are substantially destabilizing $(\Delta \Delta \mathrm{G}>1.5 \mathrm{kcal} / \mathrm{mol})^{32}$. These include Asp95 and Ser101 on CaM $\mathrm{C}_{\mathrm{C}}$ and K192 and D208 on the eEF-2K $\mathrm{K}_{\mathrm{TR}} \mathrm{KD}$, whose side-chains form a mesh of hydrogen bonds centered on $\mathrm{K} 192(\Delta \Delta \mathrm{G}=2.5 \mathrm{kcal} / \mathrm{mol})$. Other notable interactions at the interface include a hydrogen bond between the Arg90 side-chain and the A164 main-chain and an L281-His107 methyl- $\pi$ interaction. These interactions collectively localize the CTM-tethered $\mathrm{CaM}_{\mathrm{C}}$ behind the kinase active site. An additional salt bridge between Asp122 and R351 couples $\mathrm{CaM}_{\mathrm{C}}$ to the R-loop.

$\mathrm{CaM}_{\mathrm{N}}$ engages $p$ eEF-2 $\mathrm{K}_{\mathrm{TR}}$ in a $\mathrm{Mg}^{2+}$-bound "closed" state 33 contrasting its $\mathrm{Ca}^{2+}$-bound “open" conformation seen in the CTM-pep complex (Extended Data Fig. 6a,b) ${ }^{31}$. To the best of our knowledge, this conformation has not been previously observed in a CaM complex. In this configuration, the $\mathrm{KD}_{\mathrm{N}} \beta 6$ - $\beta$ 7-loop associates with shallow hydrophobic grooves on $\mathrm{CaM}_{\mathrm{N}}$; F155 interacts with Leu39 and Phe12, and L156 with Leu4 and Met76 (Fig. 3d). CaM N $_{\mathrm{N}}$ is further stabilized through numerous crystal contacts with symmetry-related neighbors (Extended Data Fig. 6c). Given this unique conformation and the high $\mathrm{Mg}^{2+}$ concentration for crystallization, we tested the influence of $\mathrm{Mg}^{2+}$ on $\mathrm{CaM}_{\mathrm{N}} / p \mathrm{eEF}-2 \mathrm{~K}_{\mathrm{TR}}$ interactions in solution. While the concentration of free $\mathrm{Mg}^{2+}$ in mammalian cells remains relatively constant at $\sim 1 \mathrm{mM}^{34}$, the $\mathrm{Ca}^{2+}$ concentration, $\sim 50-100 \mathrm{nM}$ in resting cells, can be enhanced to $\sim 100 \mu \mathrm{M}^{35}$ in calcium-microdomains under 
specific stimuli. The $\mathrm{Ca}^{2+}: \mathrm{Mg}^{2+}$ ratio (1:100) used for crystallization lies well within this cellular range. eEF-2K $\mathrm{K}_{\mathrm{TR}}$ retains the ability to efficiently auto-phosphorylate on $\mathrm{T} 348$ under these conditions (Extended Data Fig. 6d). NMR analyses (Extended Data Fig. 7) using Ile- $\delta 1$ and Met$\varepsilon$ resonances of $\mathrm{CaM}$ indicate no appreciable perturbations in the $\mathrm{CaM}_{\mathrm{C}}$ resonances with increasing $\mathrm{Mg}^{2+}$ within a complex formed in the presence of $\mathrm{Ca}^{2+}$. This suggests that $\mathrm{Mg}^{2+}$, even at very high concentrations, is unable to affect the interactions of peEF-2K $K_{\mathrm{TR}}$ with $\mathrm{CaM}_{\mathrm{C}}$ significantly. In contrast, $\mathrm{CaM}_{\mathrm{N}}$ resonances display evidence of exchange between $\mathrm{Ca}^{2+}$ - and $\mathrm{Mg}^{2+}$-bound conformations with increasing $\mathrm{Mg}^{2+}$. Indeed, at very high $\mathrm{Mg}^{2+}$ concentration where the $\mathrm{Ca}^{2+}: \mathrm{Mg}^{2+}$ ratio is heavily skewed towards $\mathrm{Mg}^{2+}$, as is the case in resting cells, $\mathrm{CaM}_{\mathrm{N}}$ disengages from $p$ eEF$2 \mathrm{~K}_{\mathrm{TR}}$.

As noted earlier, the $\mathrm{CaM}_{\mathrm{C}}$ metal-binding sites also appear to be occupied by $\mathrm{Ca}^{2+}$ in the $\mathrm{CaM} \bullet p \mathrm{eEF}-2 \mathrm{~K}_{\mathrm{TR}}$ complex, contrasting our previous studies on the CaM•CTM-pep ${ }^{31}$ and $\mathrm{CaM} \cdot \mathrm{eEF}-2 \mathrm{~K}_{\mathrm{TR}}{ }^{36}$ complexes, both carried out in the absence of $\mathrm{Mg}^{2+}$, using high- and lowresolution NMR approaches, respectively. Given the similar open configuration of $\mathrm{CaM}_{\mathrm{C}}$ in the complexes with peEF-2K $\mathrm{K}_{\mathrm{TR}}$ and CTM-pep, it is apparent that minor rearrangements of its $\mathrm{Ca}^{2+}$ binding EF-hands would suffice to accommodate $\mathrm{Ca}^{2+}$. Indeed, a difference in the engagement of $\mathrm{CaM}_{\mathrm{C}}$ by the CTM (a shift of approximately half a helical turn) is seen upon comparing the two cases; the small accompanying changes in the corresponding EF-hands appear sufficient to accommodate $\mathrm{Ca}^{2+}$ (Extended Data Fig. 8).

It has been shown that the maximal activity of eEF-2K towards a peptide substrate is independent of $\mathrm{Ca}^{2+}$ with similar $k_{\text {cat }}$ values obtained in its absence $\left(11 \mathrm{sec}^{-1}\right)$ or presence $\left(25 \mathrm{sec}^{-}\right.$ ${ }^{1}$ ), contrasting a $\sim 900$-fold increase in the apparent $\mathrm{Ca}^{2+}$-induced CaM-affinity (37 $\mu \mathrm{M}$ versus 42 $\mathrm{nM})^{37}$. The similar $k_{\text {cat }}$ values suggest that divalent cations $\left(\mathrm{Mg}^{2+}\right.$ or $\left.\mathrm{Ca}^{2+}\right)$ within $\mathrm{CaM}$ minimally 
influence the nature of the active complex. Instead, the predominant role of these ions is to modulate the overall affinity of the CaM/eEF-2K $\mathrm{K}_{\mathrm{TR}}$ interactions, i.e., to alter the "concentration" of the complex through additional interactions largely independent of those that define the active state.

\section{Coupling of $\mathrm{CaM}_{\mathrm{C}}$ to the kinase active-site}

The loop (regulatory element, RE) that connects the eEF-2K $\mathrm{K}_{\mathrm{TR}} \mathrm{CTM}$ and KD contains a 96 PDPWA $\stackrel{100}{ }$ sequence, fully conserved in vertebrates ( ${ }^{97} \mathrm{DPW} \underline{99}$ is universally conserved), that has been deemed essential for activity ${ }^{38}$. In the CaM•peEF-2K $\mathrm{K}_{\mathrm{TR}}$ complex, D97, aligned through the flanking P96 and P98, hydrogen-bonds to the main-chains of W99 and A100 using its side-chain and main-chain, respectively. This conformation generates a bulge within the RE, placing W99 within a pocket created by the side-chains of P98, F102, F138, R148, V168, and Y231 (Fig. 4a). This configuration aligns a "spine" (invoking Kornev et al. ${ }^{39}$ ) comprising of $\mathrm{CaM}_{\mathrm{C}}$ and highlyconserved, predominantly hydrophobic (except R148) residues of the CTM, RE, and $\mathrm{KD}_{\mathrm{N}}$ to support the energetic coupling of CaM-binding to the active site through the bound ATP (Fig. 4b).

Given the close-packed nature of the spine in the CaM•peEF-2 $\mathrm{K}_{\mathrm{TR}}$ complex, one may predict that its disruption would have profound functional consequences. For example, a W99A mutation is expected to be substantially more perturbative than replacement by a similarly sized Leu, as borne out by functional studies ${ }^{40}$. Further, P98 hydroxylation ${ }^{38}$ or a His 107 Lys mutation ${ }^{20}$, both of which would significantly misalign the spine, have been shown to be deleterious to function. This effect contrasts a more modest impact of a less disruptive His 107Ala mutation ${ }^{20}$. Additionally, sequence analyses suggest that mutations in the least, but still significantly conserved, spine residues, F138 and R148, co-vary with a nearby catalytic residue, R144. These are P139/P149/V145 in Ursus americanus and V137/V147/K143 in Bos mutus, perhaps resulting 
in non-functional enzymes. Thus, in our proposed model, W85 provides intrinsic binding energy ${ }^{41}$ for the interaction with $\mathrm{CaM}_{\mathrm{C}}{ }^{31}$, which is utilized, in part, to stabilize the configuration of the spine and concomitantly the active state of the kinase; W99 only contributes to the latter effect. This mechanism is consistent with the observation that a W85A mutation leads to an $\sim 9$-fold reduction in the catalytic activity and an $\sim 8$-fold decrease in the CaM-affinity; a W99A mutation leads to a $\sim 30$-fold decrease in the catalytic activity without affecting CaM-affinity (Fig. 4c).

\section{Conclusions and outlook}

Our structure of the $\mathrm{CaM} \bullet p \mathrm{EF}-2 \mathrm{~K}_{\mathrm{TR}}$ complex provides a framework to interpret the large body of biochemical and functional data generated by almost four decades of work on this unique enzyme in atomic detail. This structure, combined with current and previous biochemical/biophysical measurements, suggests a central role of $\mathrm{CaM}_{\mathrm{C}}$ in activating eEF-2K and defining the nature of the active state. In contrast, $\mathrm{CaM}_{\mathrm{N}}$ has a more supporting role, serving primarily as a sensor of dynamic changes in $\mathrm{Ca}^{2+}$, e.g., during neuronal $\mathrm{Ca}^{2+}$ spikes/waves, in the face of a relatively constant $\mathrm{Mg}^{2+}$ level, to enhance the cellular concentration of active complex. This regulation ensures maintenance of basal levels of the active species and optimal elongation rates through eEF-2 phosphorylation under normal cellular conditions while allowing its rapid modulation in response to specific signals.

However, the current structure leaves several unresolved questions about eEF-2K regulation. One such puzzle concerns the mechanism of the stimulatory auto-phosphorylation on $\mathrm{S} 500^{42}$, which is also a target of protein kinase A $(\mathrm{PKA})^{43}$. S500 is buried in a shallow pocket lined by the side-chains of H260, E264, H268, and F309 on the face opposite key catalytic elements, more than $20 \AA$ away from the base D274 (Extended Data Fig. 9a). Auto-phosphorylation in cis could, in principle, be achieved by a partial unfolding of $\alpha 1^{\prime}$ ' with the added flexibility provided 
by an intact R-loop in full-length eEF-2K. Indeed, auto-phosphorylation on S500 is extremely slow and requires prior phosphorylation on $\mathrm{T} 348^{37}$; S500 phosphorylation is not detected in $p$ eEF-2K $\mathrm{K}_{\mathrm{TR}}$. Alternatively, auto-phosphorylation in trans (or phosphorylation by PKA) would require less substantial structural transitions and occur within a transiently assembled 2:2 species with an antiparallel arrangement of its constituent CaM•peEF-2K $\mathrm{K}_{\mathrm{TR}}$ subunits. Such an assembly would be compatible with our previous crosslinking MS (XLMS) results ${ }^{22}$, which are not consistent with the current heterodimeric structure (Extended Data Fig. 9b), without invoking dramatic conformational changes. Native MS studies on the CaM•eEF-2K $\mathrm{K}_{\mathrm{TR}}$ complex reveal charge states corresponding to a 2:2 complex, albeit at extremely low levels ${ }^{22}$. Similar questions regarding the conformational changes that enable (or follow) other regulatory phosphorylation events, e.g., on S366 (a target for $\mathrm{p} 90^{\mathrm{RSK} 44}$ ), also remain unresolved since several of these modulatory R-loop sites are missing in eEF-2 $\mathrm{K}_{\mathrm{TR}}$.

Acknowledgments: The authors thank Mr. Fatlum Hajredini and Dr. Kwangwoon Lee (Harvard Medical School) for their comments about the work. Dr. Kevin Battaile (New York Structural Biology Center) is thanked for his support during data collection and analysis.

Funding: This work is supported by NIH awards R01 GM123252 (to KND and RG) and the Welch Foundation F-1390 (KND). The National Crystallization Center at Hauptman-Woodward Medical Research Institute is supported through NIH award R24 GM141256. Use of the NYX beamline (19-ID) at the National Synchrotron Light Source II (NSLS II) is supported by the member institutions of the New York Structural Biology Center. NSLS II is a United States Department of Energy (DOE) Office of Science User Facility operated for the DOE Office of Science by Brookhaven National Laboratory under Contract DE-SC0012704. 
Author Contributions: AP prepared samples for all biophysical measurements and optimized crystallization conditions together with EAI; AP and EAI collected crystallographic data; AP calculated and refined the structural model with support from EAI and DJ; AP performed and analyzed all the solution NMR measurements; KL, AB, and EAK performed the functional studies; NW designed the eEF-2K $\mathrm{K}_{\mathrm{TR}}$ construct; KND and RG developed the project; AP generated the first draft of the manuscript and figures that KND and RG subsequently refined with input from all the authors.

Data availability: Atomic coordinates for the CaM•peEF-2 $\mathrm{K}_{\mathrm{TR}}$ complex have been deposited in the Protein Data Bank (PDB) with accession code 7SHQ.

Competing interests: The authors declare no competing interests. 


\section{References}

1 Buttgereit, F. \& Brand, M. D. A hierarchy of ATP-consuming processes in mammalian cells. Biochem. J. 312, 163-167 (1995).

2 Stein, K. C. \& Frydman, J. The stop-and-go traffic regulating protein biogenesis: How translation kinetics controls proteostasis. J. Biol. Chem. 294, 2076-2084 (2019).

3 Ryazanov, A. G., Shestakova, E. A. \& Natapov, P. G. Phosphorylation of elongation factor 2 by EF-2 kinase affects rate of translation. Nature 334, 170-173 (1988).

4 Ryazanov, A. G. \& Davydova, E. K. Mechanism of elongation factor 2 (EF-2) inactivation upon phosphorylation. Phosphorylated EF-2 is unable to catalyze translocation. FEBS Lett. 251, 187-190 (1989).

5 Tavares, C. D. et al. The molecular mechanism of eukaryotic elongation factor 2 kinase activation. J. Biol. Chem. 289, 23901-23916 (2014).

6 Swulius, M. T. \& Waxham, M. N. $\mathrm{Ca}^{2+} /$ calmodulin-dependent protein kinases. Cell. Mol. Life Sci. 65, 2637-2657 (2008).

7 Liu, Y., Beyer, A. \& Aebersold, R. On the dependency of cellular protein levels on mRNA abundance. Cell 165, 535-550 (2016).

8 Schuller, A. P. \& Green, R. Roadblocks and resolutions in eukaryotic translation. Nat. Rev. Mol. Cel.l Biol. 19, 526-541 (2018).

9 Han, N. C., Kelly, P. \& Ibba, M. Translational quality control and reprogramming during stress adaptation. Exp. Cell Res. 394, 112161 (2020).

10 Middelbeek, J., Clark, K., Venselaar, H., Huynen, M. A. \& van Leeuwen, F. N. The alphakinase family: an exceptional branch on the protein kinase tree. Cell. Mol. Life Sci. 67, 875890 (2010). 
11 Nairn, A. C., Bhagat, B. \& Palfrey, H. C. Identification of calmodulin-dependent protein kinase III and its major Mr 100,000 substrate in mammalian tissues. Proc. Natl. Acad. Sci. USA 82, 7939-7943 (1985).

12 Nairn, A. C. \& Palfrey, H. C. Identification of the major $M_{r} 100,000$ substrate for calmodulin-dependent protein kinase III in mammalian cells as elongation factor-2. J. Biol. Chem. 262, 17299-17303 (1987).

13 Yang, W., Zhou, X., Ryazanov, A. G. \& Ma, T. Suppression of the kinase for elongation factor 2 alleviates mGluR-LTD impairments in a mouse model of Alzheimer's disease. Neurobiol. Aging 98, 225-230 (2021).

14 Jan, A. et al. Activity of translation regulator eukaryotic elongation factor-2 kinase is increased in Parkinson disease brain and its inhibition reduces alpha synuclein toxicity. Acta Neuropathol. Commun. 6, 54 (2018).

15 Leprivier, G. et al. The eEF2 kinase confers resistance to nutrient deprivation by blocking translation elongation. Cell 153, 1064-1079 (2013).

16 Xie, J. et al. Eukaryotic elongation factor 2 kinase upregulates the expression of proteins implicated in cell migration and cancer cell metastasis. Int. J. Cancer 142, 1865-1877 (2018).

17 Temme, L. \& Asquith, C. R. M. eEF2K: an atypical kinase target for cancer. Nat. Rev. Drug Discov. 20, 577 (2021).

18 Beretta, S., Gritti, L., Verpelli, C. \& Sala, C. Eukaryotic elongation factor 2 kinase a pharmacological target to regulate protein translation dysfunction in neurological diseases. Neuroscience 445, 42-49 (2020). 
19 Proud, C. G. Regulation and roles of elongation factor 2 kinase. Biochem. Soc. Trans. 43, 328-332 (2015).

20 Xie, J. et al. Molecular mechanism for the control of eukaryotic elongation factor 2 kinase by pH: role in cancer cell survival. Mol. Cell Biol. 35, 1805-1824 (2015).

21 Redpath, N. T., Price, N. T., Severinov, K. V. \& Proud, C. G. Regulation of elongation factor-2 by multisite phosphorylation. Eur. J. Biochem. 213, 689-699 (1993).

22 Will, N. et al. Structural dynamics of the activation of elongation factor 2 kinase by $\mathrm{Ca}^{2+}-$ calmodulin. J. Mol. Biol. 430, 2802-2821 (2018).

23 Yamaguchi, H., Matsushita, M., Nairn, A. C. \& Kuriyan, J. Crystal structure of the atypical protein kinase domain of a TRP channel with phosphotransferase activity. Mol. Cell 7, 1047-1057 (2001).

24 Ye, Q., Crawley, S. W., Yang, Y., Cote, G. P. \& Jia, Z. Crystal structure of the alpha-kinase domain of Dictyostelium myosin heavy chain kinase A. Sci. Signal. 3, ra17 (2010).

25 Ye, Q. et al. Structure of the Dictyostelium myosin-II heavy chain kinase A (MHCK-A) alpha-kinase domain apoenzyme reveals a novel autoinhibited conformation. Sci. Rep. 6 (2016).

26 Pigott, C. R. et al. Insights into the regulation of eukaryotic elongation factor 2 kinase and the interplay between its domains. Biochem. J. 442, 105-118 (2012).

27 Piserchio, A. et al. Solution structure of the carboxy-terminal tandem repeat domain of eukaryotic elongation factor 2 kinase and its role in substrate recognition. J. Mol. Biol. 431, 2700-2717 (2019).

28 Mittl, P. R. \& Schneider-Brachert, W. Sel1-like repeat proteins in signal transduction. Cell Signal 19, 20-31 (2007). 
29 Iyer, A. H., Krishna Deepak, R. N. V. \& Sankararamakrishnan, R. Imidazole nitrogens of two histidine residues participating in $\mathrm{n}-\mathrm{h} . . . \mathrm{n}$ hydrogen bonds in protein structures: structural bioinformatics approach combined with quantum chemical calculations. J. Phys. Chem. B 122, 1205-1212(2018).

30 Piserchio, A. et al. Structural dynamics of the complex of calmodulin with a minimal functional construct of eukaryotic elongation factor 2 kinase and the role of Thr348 autophosphorylation. Protein Sci. 30, 1221-1234 (2021).

31 Lee, K. et al. Structural basis for the recognition of eukaryotic elongation factor 2 kinase by calmodulin. Structure 24, 1441-1451 (2016).

32 Kortemme, T., Kim, D. E. \& Baker, D. Computational alanine scanning of protein-protein interfaces. Sci. STKE 2004, p12 (2004).

33 Senguen, F. T. \& Grabarek, Z. X-ray structures of magnesium and manganese complexes with the N-terminal domain of calmodulin: insights into the mechanism and specificity of metal ion binding to an EF-hand. Biochemistry 51, 6182-6194 (2012).

34 Romani, A. Regulation of magnesium homeostasis and transport in mammalian cells. Arch. Biochem. Biophys. 458, 90-102 (2007).

35 Fernandez-Sanz, C., De la Fuente, S. \& Sheu, S. S. Mitochondrial $\mathrm{Ca}^{2+}$ concentrations in live cells: quantification methods and discrepancies. FEBS Lett. 593, 1528-1541 (2019).

36 Lee, K., Kumar, E. A., Dalby, K. N. \& Ghose, R. The role of calcium in the interaction between calmodulin and a minimal functional construct of eukaryotic elongation factor 2 kinase. Protein Sci. 28, 2089-2098 (2019).

37 Tavares, C. D. J. et al. Signal integration at elongation factor 2 kinase: the roles of calcium, calmodulin, and Ser-500 phosphorylation. J. Biol. Chem. 292, 2032-2045 (2017). 
38 Moore, C. E. et al. Elongation factor 2 kinase is regulated by proline hydroxylation and protects cells during hypoxia. Mol. Cell. Biol. 35, 1788-1804 (2015).

39 Kornev, A. P., Taylor, S. S. \& Ten Eyck, L. F. A helix scaffold for the assembly of active protein kinases. Proc. Natl. Acad. Sci. USA 105, 14377-14382 (2008).

40 Moore, C. E., Regufe da Mota, S., Mikolajek, H. \& Proud, C. G. A conserved loop in the catalytic domain of eukaryotic elongation factor 2 kinase plays a key role in its substrate specificity. Mol. Cell Biol. 34, 2294-2307 (2014).

41 Jencks, W. P. On the attribution and additivity of binding ennergies. Proc. Natl. Acad. Sci. USA 78, 4046-4050 (1981).

42 Tavares, C. D. et al. Calcium/calmodulin stimulates the autophosphorylation of elongation factor 2 kinase on Thr-348 and Ser-500 to regulate its activity and calcium dependence. Biochemistry 51, 2232-2245 (2012).

43 Redpath, N. T. \& Proud, C. G. Cyclic AMP-dependent protein kinase phosphorylates rabbit reticulocyte elongation factor-2 kinase and induces calcium-independent activity. Biochem, J, 293, 31-34 (1993).

44 Wang, X. et al. Regulation of elongation factor 2 kinase by p90 $0^{\mathrm{RSK} 1}$ and p70 S6 kinase. EMBO J. 20, 4370-4379 (2001). 


\section{Methods}

\section{Crystallization of the CaM•pTR complex}

The protocols used for the expression and purification of eEF-2 $\mathrm{K}_{\mathrm{TR}}$ and $\mathrm{CaM}$, the selective phosphorylation of the former at $\mathrm{T} 348$ (to generate $p \mathrm{eEF}-2 \mathrm{~K}_{\mathrm{TR}}$ ), and the subsequent purification of the 1:1 heterodimeric CaM•peEF-2K $\mathrm{K}_{\mathrm{TR}}$ complex were performed as described previously ${ }^{22,30}$. The final samples used in the crystallization trials consisted of $\sim 11.0 \mathrm{mg} / \mathrm{mL}$ of the CaM•peEF$2 \mathrm{~K}_{\mathrm{TR}}$ complex in a buffer containing $20 \mathrm{mM}$ Tris $(\mathrm{pH} 7.5), 100 \mathrm{mM} \mathrm{NaCl}, 3.0 \mathrm{mM} \mathrm{CaCl}, 1.0 \mathrm{mM}$ tris(2-carboxyethyl)phosphine (TCEP, GoldBio), $1.5 \mathrm{mM} \mathrm{MgCl}_{2}$ and $1.0 \mathrm{mM}$ AMP-PNP (Millipore Sigma). Initial screens to identify crystallization conditions were performed under oil at the National Crystallization Center at the Hauptman-Woodward Medical Research Institute. Potential conditions were further optimized in-house, and optimal conditions were identified as the following: $100 \mathrm{mM}$ Bis-Tris ( $\mathrm{pH}$ 6.9), 200-300 $\mathrm{mM} \mathrm{MgCl}_{2}$, and 20-26\% PEG3350 combined in a 1:1 or 2:1 ratio plated on Greiner 72-well micro-batch plates (Hampton Research) under paraffin oil (EMD Chemicals) at ambient temperature. Multiple crystal clusters emerged in less than 12 hours, reaching their maximum size within a few days. Most of these, however, were extremely fragile and diffracted poorly. A single condition, 1:1 ratio, 24\% PEG3350, and $300 \mathrm{mM}$ $\mathrm{MgCl}_{2}$, yielded crystals that produced diffraction data of good quality.

\section{Structure determination}

Data were collected at the NSLS-II light source at Brookhaven National Labs utilizing the 19-ID (NYX) beamline. Two datasets collected on distinct regions of the same crystal were processed and combined using the autoPROC toolbox ${ }^{45}$ resulting in a dataset with a resolution of $2.3 \AA$. The crystal $\left(\mathrm{P} 3{ }_{1} 21\right)$ with an elongated unit cell $(\mathrm{a}=\mathrm{b}=58.49 \AA, \mathrm{c}=365.78 \AA)$ contained $\mathrm{a}$ single $\mathrm{CaM} \bullet p \mathrm{EF}-2 \mathrm{~K}_{\mathrm{TR}}$ heterodimer in the asymmetric unit. Initial phases were obtained using 
phemix.mr_rosetta ${ }^{46}$ and an alignment file obtained from the HHpred server ${ }^{47}$ containing 53 entries, including sequences corresponding to the crystal structures of the $\alpha$-kinase domains of MHCK-II ${ }^{25}$, ChaK ${ }^{23}$, together with those of several SEL-1 and TPR repeat-containing proteins. The initial model was built using multiple cycles consisting of Phaser ${ }^{48}$, $\operatorname{Coot}^{49}$, and phenix.refine ${ }^{50}$. At the end of the initial build, further optimization was carried out using the PDB_REDO server ${ }^{51}$, yielding a model that contained 469 eEF-2K $\mathrm{K}_{\mathrm{TR}}$ residues $(88 \%)$ and the Clobe of CaM. Most of the CaM N-lobe (except the loop between helices $\alpha_{C}$ and $\alpha_{D}$ ) was built through iterative manual fitting with Coot followed by refinement using Phenix. A comparison of the CaM N-lobe fold with existing CaM structures using the DALI server ${ }^{52}$ showed its close resemblance to a $\mathrm{Mg}^{2+}$-bound closed form. Indeed, molecular replacement using Phaser and PDB:3UCW ${ }^{33}$ together with the rest of the CaM•eEF-2K $\mathrm{K}_{\mathrm{TR}}$ complex structure yielded a nearly complete model. The remaining missing segments of the structure of the complex were then progressively added and improved using Coot, phenix.refine, and ISoLDE ${ }^{53}$. Cations were identified using the CheckMyBlob server ${ }^{54}$ and inserted with solvent molecules using Coot and refined using Phenix. The final structural model consisted of 493 and 145 residues of eEF-2K $\mathrm{K}_{\mathrm{TR}}$ $(92.8 \%)$ and $\mathrm{CaM}(97.9 \%)$, respectively, together with $1 \mathrm{Zn}^{2+}, 2 \mathrm{Ca}^{2+}, 5 \mathrm{Mg}^{2+}$ ions and 145 water molecules. No discernible density for AMPPNP was observed. Details about data collection and refinement are shown in Extended Data Table 1.

The sequence conservation in eEF- $2 \mathrm{~K}_{\mathrm{TR}}$ was obtained using the ConSurf server ${ }^{55}$ with default parameters that generated a comparison with 150 non-redundant sequences $(95 \%$ identity cut-off). The generated alignment file was read into UCSF ChimeraX ${ }^{56}$ and represented on the eEF-2K $\mathrm{K}_{\mathrm{TR}}$ structure with the corresponding residues colored by their AL2CO scores ${ }^{57}$. 


\section{Solution NMR spectroscopy}

${ }^{13} \mathrm{C},{ }^{1} \mathrm{H}-\mathrm{Ile}-\delta 1$, Met- $\varepsilon,{ }^{2} \mathrm{H},{ }^{15} \mathrm{~N}$-labeled CaM (IM-labeled CaM) ${ }^{36}$ and unlabeled $p$ eEF-2K $\mathrm{KR}^{30}$ were prepared as previously described, and the corresponding 1:1 heterodimeric complex was purified as described above. All NMR samples (unless specifically noted) were prepared in a buffer (NMR buffer) containing $20 \mathrm{mM}$ HEPES (pH 7.5), $100 \mathrm{mM} \mathrm{NaCl}, 1.0 \mathrm{mM}$ TCEP, and 5\% ${ }^{2} \mathrm{H}_{2} \mathrm{O}$. The following samples were prepared: (1) IM-labeled CaM alone $(\sim 100 \mu \mathrm{M})$ in NMR buffer containing $3.0 \mathrm{mM} \mathrm{CaCl} 2$ or (2) as part of the corresponding $\mathrm{CaM} \bullet p \mathrm{eEF}-2 \mathrm{~K}_{\mathrm{TR}}$ complex $(\sim 147$ $\mu \mathrm{M})$; (3) IM-labeled CaM $(\sim 100 \mu \mathrm{M})$ in NMR buffer containing $5.0 \mathrm{mM}$ EGTA and $310 \mathrm{mM}$ $\mathrm{MgCl}_{2}$; (4) IM-labeled CaM alone $(\sim 100 \mu \mathrm{M})$ in NMR buffer containing $3.0 \mathrm{mM} \mathrm{CaCl}$ and 300 $\mathrm{mM} \mathrm{MgCl} 2$ or $(5)$ as part of the corresponding CaM•peEF-2K $\mathrm{K}_{\mathrm{TR}}$ complex $(\sim 147 \mu \mathrm{M}) ;(6) \sim 50 \mu \mathrm{M}$ samples of IM-labeled CaM in NMR buffer containing $\sim 150 \mu \mathrm{M}$ DSS and 0, 0.2, 0.4, 5.0, 20, 103 or $310 \mathrm{mM} \mathrm{MgCl}_{2}$ (all samples contained EGTA in a $\sim 1 / 60$ ratio with respect to $\mathrm{Mg}^{2+}$ ); (7) samples containing $\sim 18 \mu \mathrm{M}$ of the CaM•peEF-2K $\mathrm{K}_{\mathrm{TR}}$ complex in buffer comprising $20 \mathrm{mM}$ HEPES (pH 7.5), $10 \mathrm{mM} \mathrm{NaCl}, 0.5 \mathrm{mM}$ TCEP, $\sim 150 \mu \mathrm{M}$ DSS, and $0.3 \mathrm{mM} \mathrm{CaCl}_{2}$ with $0,1.0,3.0,6.0,12.0$, 36, 68, 115, 240 and $450 \mathrm{mM} \mathrm{MgCl} 2 .{ }^{1} \mathrm{H},{ }^{13} \mathrm{C}$ SOFAST-HMQC ${ }^{58}$ experiments were carried out on all samples using sweep-widths of 13.95 ppm (512 complex points) and $12.0 \mathrm{ppm}$ (128 complex points) in the direct and indirect dimensions, respectively. All experiments were carried out at 25 ${ }^{\circ} \mathrm{C}$ on an $800 \mathrm{MHz}$ Bruker Avance-III HD spectrometer equipped with a triple-resonance cryogenic probe capable of applying pulsed-field gradients along the z-axis. Data were processed using nmrPipe $^{59}$ and analyzed using nmrView ${ }^{60}$. Chemical shift perturbations $(\Delta \delta$ in ppm) were calculated using ${ }^{36}$

$$
\Delta \delta=\sqrt{\left(\delta_{r e f, H}-\delta_{H}\right)^{2}+\left[\frac{1}{3.94}\left(\delta_{r e f, C}-\delta_{C}\right)\right]^{2}}
$$


Where $\delta_{r e f, H}$ and $\delta_{r e f, C}$ are the reference ${ }^{1} \mathrm{H}$ and ${ }^{13} \mathrm{C}$ methyl chemical shifts, respectively, and $\delta_{H}$ and $\delta_{C}$ are the corresponding shifts in the presence of relevant additives.

\section{Small-angle X-ray scattering}

Small-angle X-ray scattering (SAXS) data were acquired on two samples containing 1.9 $\mathrm{mg} / \mathrm{mL}(\mathrm{lc})$ and $6.4 \mathrm{mg} / \mathrm{mL}(\mathrm{hc})$ of the CaM•eEF-2K $\mathrm{K}_{\mathrm{TR}}$ complex (unphosphorylated eEF-2K $\mathrm{K}_{\mathrm{TR}}$ ), as previously described $^{22}$. These data were reanalyzed using ATSAS 3.0.1 software ${ }^{61}$, and identical results as those shown in Table 3 of Will et al. ${ }^{22}$, were obtained. However, a slightly different approach was utilized to obtain ab initio 3-dimensional models from the SAXS data. 25 structures were calculated for each of the he and lc datasets using DAMMIF ${ }^{62}$ using default parameters in the slow mode and clustered using DAMCLUST ${ }^{63}$. For the he dataset, a total of 6 clusters were obtained in which 3 (hc_1, hc_2, hc_4) contained a single member, 1 each contained 2 (hc_5) or 3 (hc_3) members, and the largest cluster (hc_6; NSD to all other clusters $=1.47 \pm 0.31$ ) comprised of 17 members. The averaged envelope from 6_hc was refined against the experimental hc dataset $\left(\chi^{2}=0.9766\right)$ using DAMMIN ${ }^{64}$ (run in the slow mode using default parameters) by restricting the search volume using DAMSTART. For the lc dataset, 2 clusters contained a single member (lc_1, 1c_2), 1 each contained 3 (1c_5), 5 (1c_6), 7 (1c_3) or 8 members (1c_4). Thus, the averaged structures from the two largest clusters (lc_3 and lc_4, NSD=1.16) were separately refined against the experimental lc dataset $\left(\chi^{2}=0.7761\right.$ and $\chi^{2}=0.7767$ for lc_3 and 1c_4, respectively).

The SAXS profile was calculated from the structure of the CaM•peEF- $2 \mathrm{~K}_{\mathrm{TR}}$ complex using CRYSOL $3^{65}$ and fitted separately to the lc and he datasets yielding $\chi^{2}$ values of 1.504 and 3.217, respectively, suggesting that the former provides a somewhat better agreement with that expected from the structure. The molecular envelopes calculated from each dataset (1c_3, lc_4, hc_6) were superimposed on the crystal structure using SUPCOMB ${ }^{66}$. The structure of the CaM•peEF-2K 
complex fitted into the refined molecular envelope of the 1c_3 cluster and a comparison of the theoretical and calculated data for the lc dataset are shown in Extended Data Fig. 2.

\section{Measurement of eEF-2K $\mathrm{TR}$ activity in the presence of $\mathrm{Mg}^{2+}$}

Auto-phosphorylation of eEF-2 $\mathrm{K}_{\mathrm{TR}}(500 \mathrm{nM})$ was carried out in buffer comprising $25 \mathrm{mM}$ HEPES (pH 7.0), $2 \mathrm{mM}$ DTT, $40 \mu \mathrm{g} / \mathrm{mL}$ BSA, $50 \mathrm{mM} \mathrm{KCl}$, and $5 \mu \mathrm{M}$ CaM. The reaction was conducted under two distinct conditions - one with $15 \mathrm{mM} \mathrm{MgCl}_{2}$ and $0.15 \mathrm{mM} \mathrm{CaCl}_{2}$ and a second with $150 \mathrm{mM} \mathrm{MgCl}_{2}$ and $1.5 \mathrm{mM} \mathrm{CaCl}_{2}$. The reaction mixture was incubated at $30{ }^{\circ} \mathrm{C}$ for $10 \mathrm{~min}$, and the reaction was initiated by the addition of $500 \mu \mathrm{M}\left[\gamma^{32} \mathrm{P}\right] \mathrm{ATP}(100-1000$ c.p.m./pmol) in a final volume of $250 \mu \mathrm{L}$. Aliquots $(10 \mathrm{pmol} / 20 \mu \mathrm{L})$ of eEF-2K $\mathrm{K}_{\mathrm{TR}}$ were removed every $0,10,23,32,40,60,120$ and $300 \mathrm{~s}$ over a 5 min time period and directly added to hot SDS-PAGE sample loading buffer containing 125 mM Tris- $\mathrm{HCl}$ (pH 6.75), 20\% glycerol (v/v), $10 \%$ 2-mercaptoethanol (v/v), 4\% SDS, and $0.02 \%$ bromophenol blue) to quench the reaction. The mixture was further heated for $5 \mathrm{~min}$ at $95^{\circ} \mathrm{C}$ to ensure complete denaturation. The samples were resolved by SDS-PAGE and stained with Coomassie Brilliant Blue. Gels were exposed for $8 \mathrm{~h}$ in a phosphorimager cassette, scanned in a Typhoon 9500 imager, and analyzed using ImageJ. Autophosphorylation was quantified by drying the gels and excising the eEF-2 $\mathrm{K}_{\mathrm{TR}}$-containing segments. The radioactivity was measured with a Packard 1500 liquid scintillation analyzer.

\section{Measurement of the activity of full-length eEF-2K and mutants.}

Assays were performed in buffer containing $25 \mathrm{mM}$ HEPES, $50 \mathrm{mM} \mathrm{KCl,} 10 \mathrm{mM} \mathrm{MgCl}$, $150 \mu \mathrm{M} \mathrm{CaCl}_{2}, 20 \mu \mathrm{g} / \mathrm{mL}$ BSA, $100 \mu \mathrm{M}$ EGTA, 2 mM DTT, 0-4 $\mu \mathrm{M}$ CaM, and $10 \mu \mathrm{M}$ soxpeptide $^{67}$. The assays used $5 \mathrm{nM}, 10.5 \mathrm{nM}$, or $8.6 \mathrm{nM}$ of unphosphorylated wild-type eEF-2K, the W85A mutant, or the W99A mutant, respectively. The reaction was initiated with the addition of $1 \mathrm{mM}$ ATP. Product turnover was monitored by fluorescence (excitation at $360 \mathrm{~nm}$, emission at 
$482 \mathrm{~nm}$ ) using a Synergy H4 plate reader (BioTek). The data were analyzed using a quadratic binding isotherm to obtain $k_{\mathrm{obs} \text {,max }}$, and $K_{\mathrm{CaM}}$ values. Experiments were performed in duplicate (for wild-type) or in triplicate (for the W85A and W99A mutants). 
45 Vonrhein, C. et al. Data processing and analysis with the autoPROC toolbox. Acta Crystallogr. D Biol. Crystallogr. 67, 293-302 (2011).

46 Terwilliger, T. C. et al. phenix.mr_rosetta: molecular replacement and model rebuilding with Phenix and Rosetta. J. Struct. Funct. Genomics 13, 81-90 (2012).

47 Hildebrand, A., Remmert, M., Biegert, A. \& Soding, J. Fast and accurate automatic structure prediction with HHpred. Proteins 77, 128-132 (2009).

48 Zwart, P. H. et al. Automated structure solution with the PHENIX suite. Meth. Mol. Biol. 426, 419-435 (2008).

49 Emsley, P. \& Cowtan, K. Coot: model-building tools for molecular graphics. Acta Crystallogr. D Biol. Crystallogr. 60, 2126-2132 (2004).

50 Afonine, P. V. et al. Towards automated crystallographic structure refinement with phenix.refine. Acta Crystallogr. D Biol. Crystallogr. 68, 352-367 (2012).

51 Joosten, R. P., Long, F., Murshudov, G. N. \& Perrakis, A. The PDB_REDO server for macromolecular structure model optimization. IUCr J 1, 213-220 (2014).

52 Holm, L. \& Laakso, L. M. Dali server update. Nucleic Acids Res. 44, W351-355 (2016).

53 Reynes, C. et al. ISoLDE: a data-driven statistical method for the inference of allelic imbalance in datasets with reciprocal crosses. Bioinformatics 36, 504-513 (2020).

54 Brzezinski, D., Porebski, P. J., Kowiel, M., Macnar, J. M. \& Minor, W. Recognizing and validating ligands with CheckMyBlob. Nucleic Acids Res. 49, W86-W92 (2021).

55 Ashkenazy, H. et al. ConSurf 2016: an improved methodology to estimate and visualize evolutionary conservation in macromolecules. Nucleic Acids Res. 44, W344-350 (2016).

56 Pettersen, E. F. et al. UCSF ChimeraX: Structure visualization for researchers, educators, and developers. Protein Sci. 30, 70-82 (2021). 
57 Pei, J. \& Grishin, N. V. AL2CO: calculation of positional conservation in a protein sequence alignment. Bioinformatics 17, 700-712 (2001).

58 Amero, C. et al. Fast two-dimensional NMR spectroscopy of high molecular weight protein assemblies. J. Am. Chem. Soc. 131, 3448-3449 (2009).

59 Delaglio, F. et al. NMRPipe: a multidimensional spectral processing system based on UNIX pipes. J. Biomo.l NMR 6, 277-293 (1995).

60 Johnson, B. A. From raw data to protein backbone chemical shifts using NMRFx processing and NMRViewJ analysis. Meth. Mol. Biol. 1688, 257-310 (2018).

61 Manalastas-Cantos, K. et al. ATSAS 3.0: expanded functionality and new tools for smallangle scattering data analysis. J. Appl. Crystallogr. 54, 343-355 (2021).

62 Franke, D. \& Svergun, D. I. DAMMIF, a program for rapid ab-initio shape determination in small-angle scattering. J. Appl. Crystallogr. 42, 342-346 (2009).

63 Petoukhov, M. V. et al. New developments in the ATSAS program package for smallangle scattering data analysis. J. Appl. Crystallogr. 45, 342-350 (2012).

64 Svergun, D. I. Restoring low resolution structure of biological macromolecules from solution scattering using simulated annealing. Biophys. J. 76, 2879-2886 (1999).

65 Franke, D. et al. ATSAS 2.8: a comprehensive data analysis suite for small-angle scattering from macromolecular solutions. J. Appl. Crystallogr. 50, 1212-1225 (2017).

66 Kozin, M. B. \& Svergun, D. I. Automated matching of high- and low-resolution structural models. J. Appl. Crystallogr. 34, 33-41 (2001).

67 Devkota, A. K. et al. High-throughput screens for eEF-2 kinase. J. Biomol. Screen. 19, 445-452 (2014). 

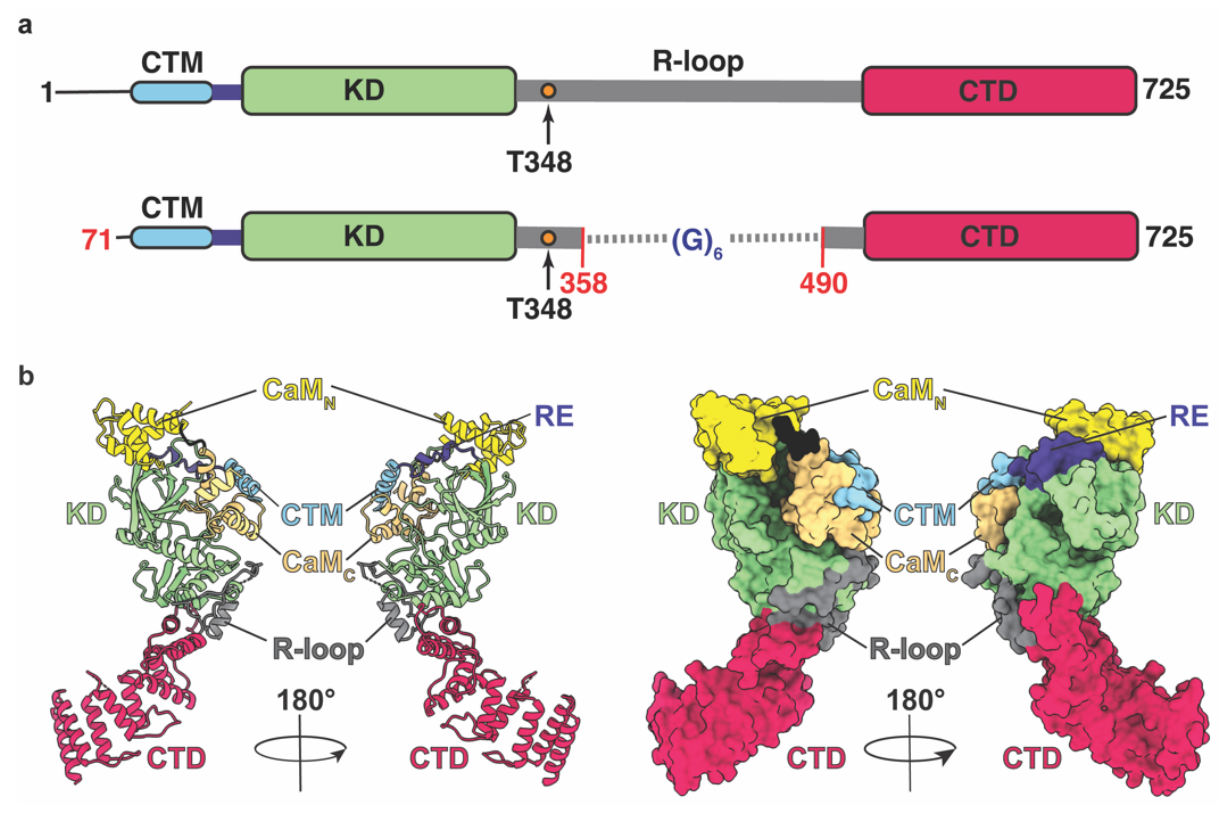

Fig. 1 Structure of the CaM॰peEF-2K $K_{\text {TR }}$ complex. a, Schematic representation of the eEF-2K (top) structural modules indicating the CTM (cyan), RE (navy-blue), KD (lime-green), R-loop (grey) and CTD (crimson). eEF-2K $\mathrm{K}_{\mathrm{TR}}$ (bottom) is missing $70 \mathrm{~N}$-terminal residues, and a 6-glycine linker replaces the 359-489 segment of the R-loop. b, Structure of the CaM•peEF-2K $\mathrm{K}_{\mathrm{TR}}$ complex shown in ribbon (left) or surface (right) representation; $\mathrm{CaM}_{\mathrm{N}}$ and $\mathrm{CaM}_{\mathrm{C}}$ are colored bright and dull yellow, respectively. The same color scheme is used in all figures unless otherwise stated. 
a

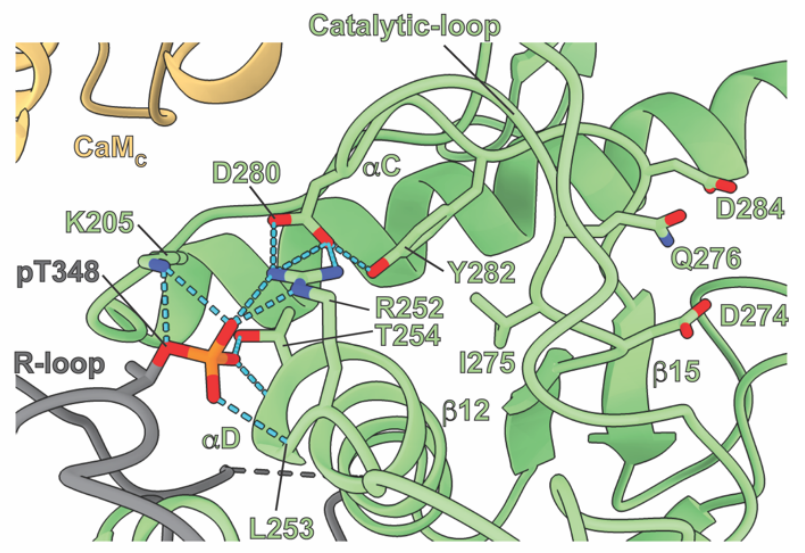

b

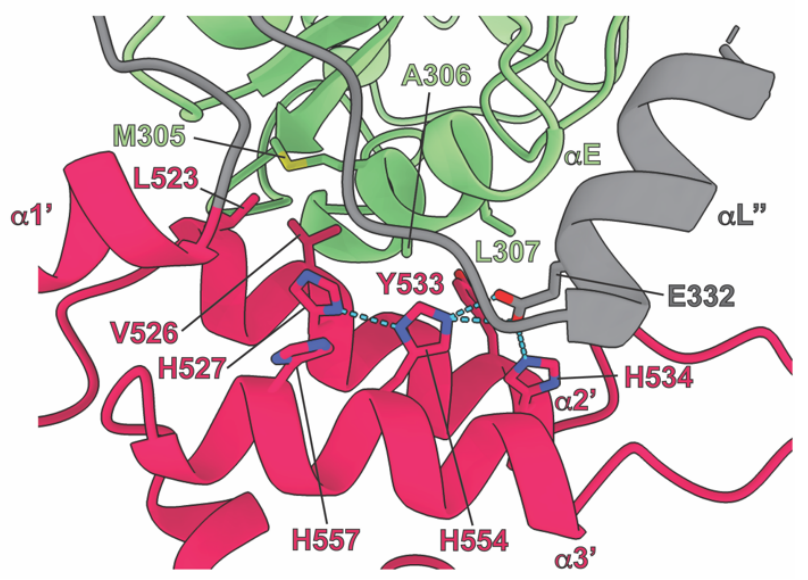

Fig. 2 Intramolecular interactions within peEF-2K $K_{T R}$ in the CaM•peEF-2K $K_{T R}$ complex. a, Interactions that stabilize $p$ T348 at the phosphate-binding pocket (PBP) and couple the PBP to the active site are indicated. Key catalytic-loop residues, including the base D274, Q276, and D284, are shown for reference. b, Key interactions involving the $\alpha E-\alpha 2$ ' $-\alpha 3$ ' element that stabilize the $\mathrm{KD} / \mathrm{CTD}$ interface are shown. 
a

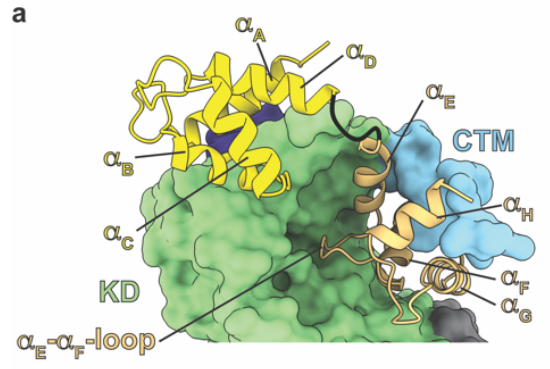

C

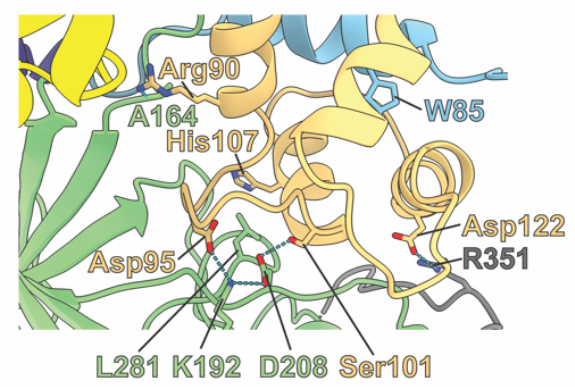

b

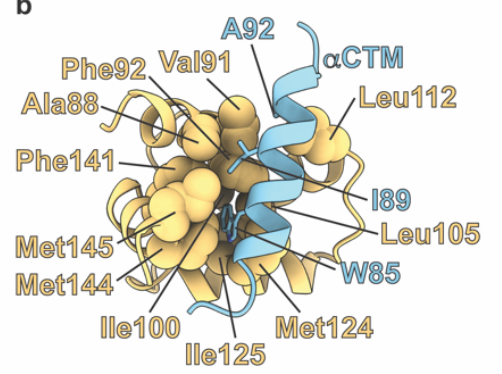

d

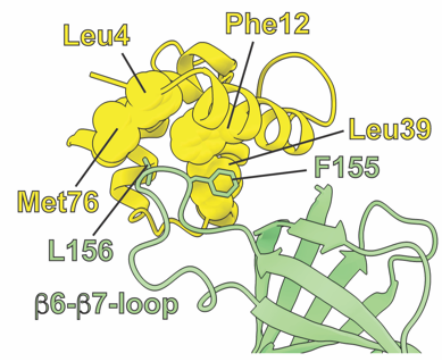

Fig. 3 Intermolecular interactions between CaM and peEF-2K $K_{T R}$ a, CaM and eEF-2K $\mathrm{K}_{\mathrm{TR}}$ are shown in ribbon and surface representations, respectively. $\mathrm{CaM}_{N}\left(\alpha_{A}-\alpha_{D}\right)$ and $\mathrm{CaM}_{C}\left(\alpha_{E}-\alpha_{G}\right)$ helices are indicated. Interactions with the hydrophobic (b) and hydrophilic (c) faces of $\mathrm{CaM}_{\mathrm{C}}$ with the CTM and $\mathrm{KD}_{\mathrm{N}}$, respectively. d, Interactions of $\mathrm{CaM}_{\mathrm{N}}$ with the $\beta 6-\beta 7-1$ oop of $\mathrm{KD}_{\mathrm{N}}$. Elements not directly involved in the interaction are hidden to aid visualization. 


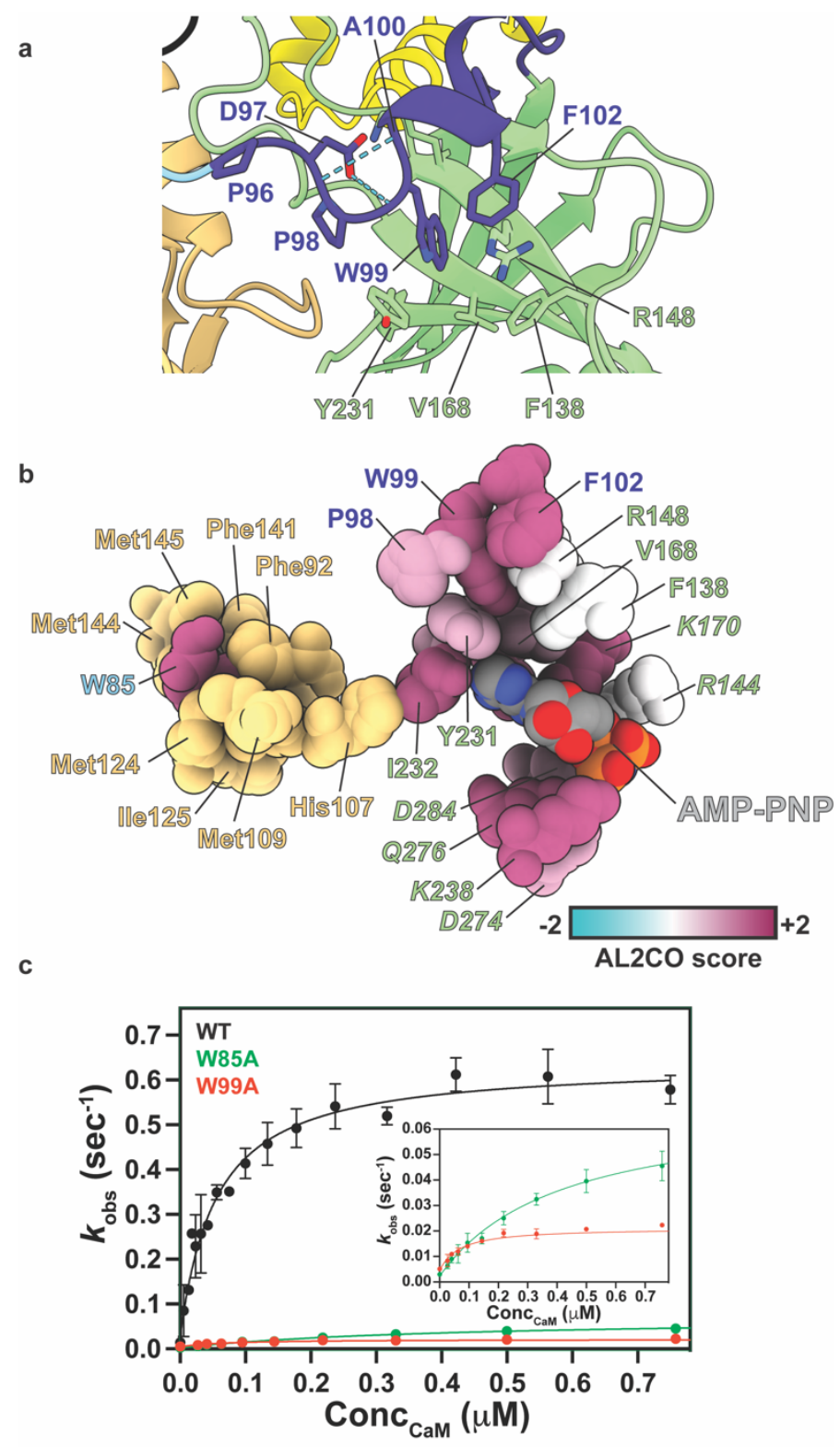

Fig. 4 Activation of eEF-2KTR by CaM. a, Interactions of the RE with $K_{\mathrm{N}}$. $\mathbf{b}$, Residues of the

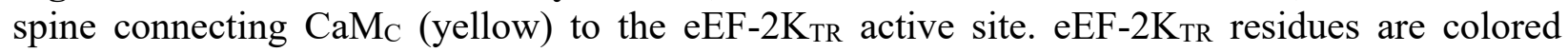
according to conservation scores (cyan: variable, maroon: fully-conserved). AMP-PNP has been modeled in. c, Activity of wild-type eEF-2K (WT, black), and corresponding W85A (green) and W99A (red) mutants. The maximal catalytic rates $\left(\mathrm{sec}^{-1}\right)$ are WT:0.61 $\pm 0.02, \mathrm{~W} 85 \mathrm{~A}: 0.07 \pm 0.002$, W99A:0.02 \pm 0.001 ; the corresponding affinities $(\mathrm{nM})$ are $52 \pm 8,406 \pm 47,62 \pm 19$. The inset shows an expansion for the two mutants. Error bars indicate standard deviations over $n=2$ (WT) and $n=3$ (mutants) measurements. 
bioRxiv preprint doi: https://doi.org/10.1101/2022.01.15.476372; this version posted January 15, 2022. The copyright holder for this preprint (which was not certified by peer review) is the author/funder. All rights reserved. No reuse allowed without permission.

\section{Extended Data and Tables}




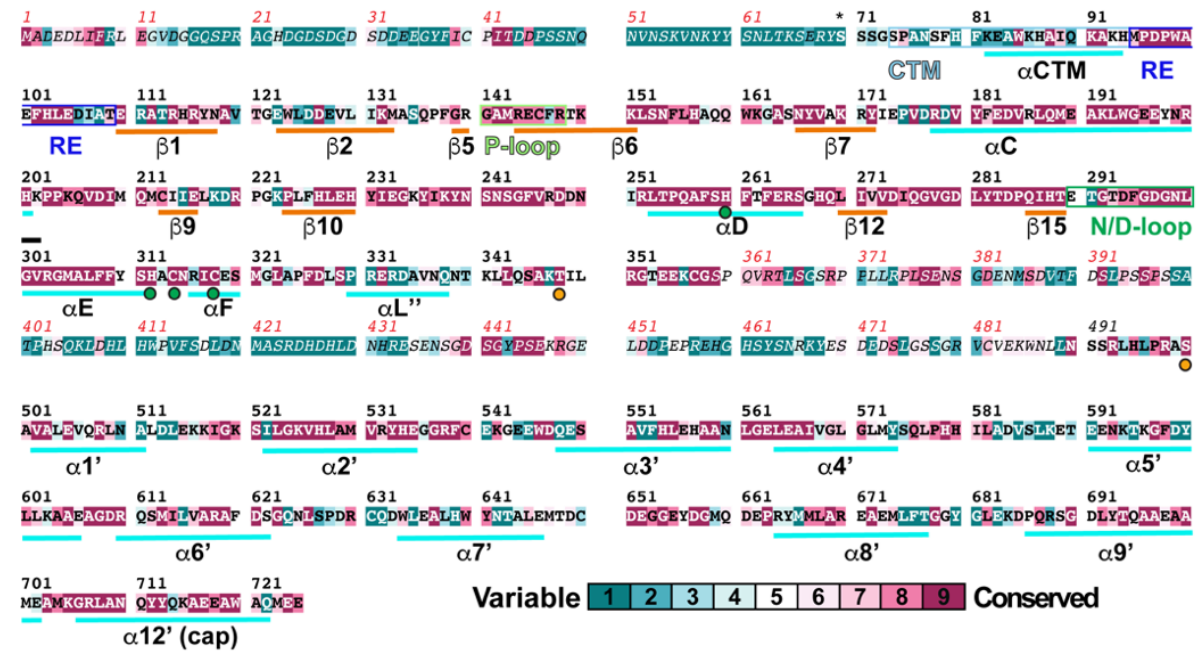

Extended Data Fig. 1 Sequence conservation in eEF-2K. Sequence conservation in eEF-2K was obtained using the ConSurf server with standard settings. Specific regions of secondary structure as determined by UCSF ChimeraX are indicated below the sequence; the location of the CaMtargeting motif (CTM, 74-95; previously known as the CaM-binding domain, CBD), regulatory element (RE, 96-109), P-loop (141-148) and N/D-loop (290-300, previously termed the G-loop), are also indicated. Labeling of the secondary structural elements for the KD (110-321) is based on that previously assigned to myosin heavy chain kinase A (MHCK-A). Green circles indicate residues $\mathrm{H} 260, \mathrm{H} 312, \mathrm{C} 314$, and $\mathrm{C} 318$ that coordinate the structural $\mathrm{Zn}^{2+}$ found in all $\alpha$-kinases. The orange circles indicate the primary (T348) and secondary (S500) auto-phosphorylation sites. The secondary structural elements for the C-terminal domain (CTD, 502-725) are primed. The 359-489 segment of the R-loop (321-501) has been replaced by a 6-glycine linker in eEF-2 $\mathrm{K}_{\mathrm{TR}}$ (previously called TR) that is also missing the first 69 residues of full-length eEF-2K; S70 (indicated by $\mathrm{a}^{\text {'*') }}$ ) that is native in eEF-2K re-appears as a remnant of cloning. The residues present or absent in the eEF- $2 \mathrm{~K}_{\mathrm{TR}}$ construct compared to full-length eEF-2K are shown in bold or italicized fonts, respectively, with the corresponding sequencing numbering indicated in black or red font. 

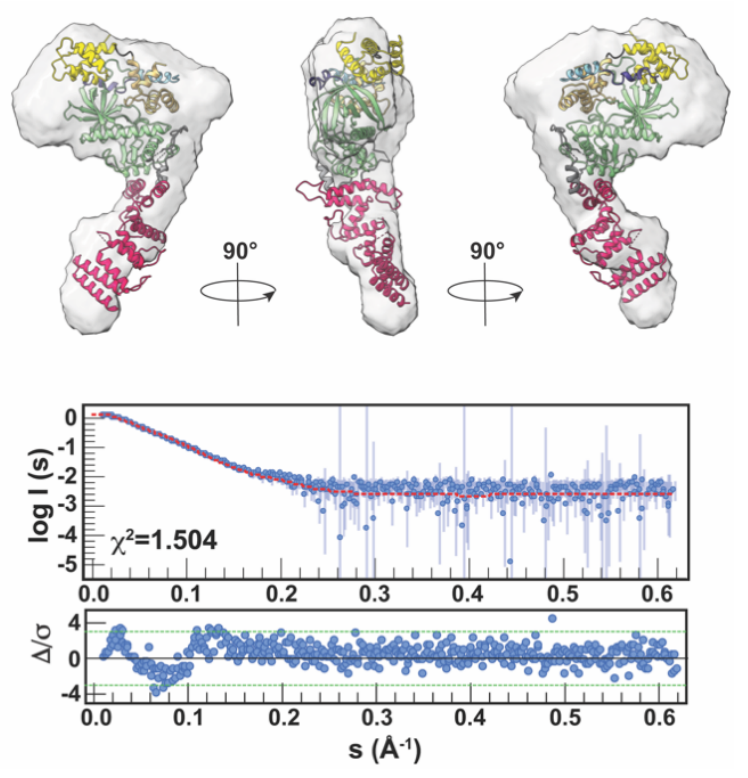

Extended Data Fig. 2 The structure of the CaM•peEF-2K $K_{T R}$ complex is consistent with solution scattering data. The structure of the CaM•peEF- $2 \mathrm{~K}_{\mathrm{TR}}$ complex fitted into the molecular envelope of one of two dominant clusters calculated from small-angle X-ray scattering (SAXS) data acquired on a low-concentration sample of the CaM•eEF-2 $\mathrm{K}_{\mathrm{TR}}$ complex (unphosphorylated T348). The corresponding experimental data (blue circles), the theoretical fit (red curve), and the reduced residuals $(\Delta / \sigma)$ are shown on the lower panel. While the SAXS-generated envelope reproduces the overall structural features in solution, the reduced residuals at low $s$ values, and the relatively large $\chi^{2}$ value, suggest differences between the molecular species. Some of these differences can be attributed to the fact that the SAXS data were acquired on the unphosphorylated complex in the presence of $\mathrm{Ca}^{2+}$ but in the absence of $\mathrm{Mg}^{2+}$. 

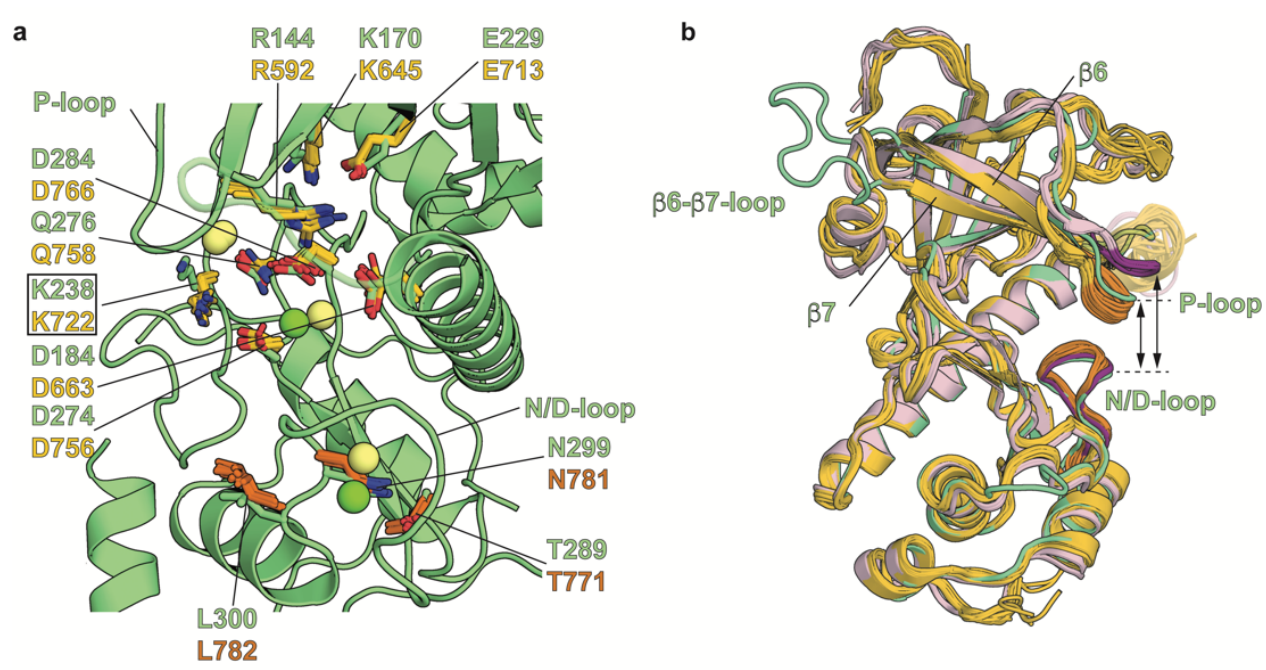

Extended Data Fig. 3 peEF-2K $K_{T R}$ is in an active state in the CaM•peEF-2K $K_{T R}$ complex. a, Comparison of the orientations of key "catalytic" residues (in green) of the eEF-2K $\mathrm{K}_{\mathrm{TR}} \mathrm{KD}$ in the $\mathrm{CaM} \cdot p \mathrm{EF}-2 \mathrm{~K}_{\mathrm{TR}}$ complex with corresponding residues (light orange) in several structures of the MHCK-A KD. R144 of eEF-2K TR (R592 in MHCK-A) coordinates the $\alpha$ - and $\beta$-phosphates of ATP; K170 (K645) engages the $\alpha$-phosphate and the adenine ring; E229 (E713) engages the adenine amino group; K238 (K722) engages the $\gamma$-phosphate of ATP; D274 (D756) is the putative catalytic base, Q758 (Q276) and D284 (D766) coordinate the catalytic $\mathrm{Mg}^{2+}$ ions and the $\gamma-$ and $\alpha / \beta$-phosphates of ATP, respectively. While no density corresponding to a nucleotide was seen in the CaM•peEF-2K $\mathrm{K}_{\mathrm{TR}}$ complex (crystallized in the presence of the slowly hydrolyzable ATP analog, AMP-PNP), all side-chains (except K238, indicated by the rectangle) are in similar orientations as their nucleotide-bound MHCK-A counterparts. The $\mathrm{Mg}^{2+}$ ions seen in the structure of the $\mathrm{CaM} \bullet p \mathrm{eEF}-2 \mathrm{~K}_{\mathrm{TR}}$ complex are shown green, and those seen in the MHCK-A structures are shown in lime-green. Also shown (MHCK-A residues in orange) are the N/D-loop residues, T289 (T771), N299 (N781), and L300 (L782). N299 is stabilized through hydrogen bonds with the T289 sidechain and the D294 main-chain. This "asparagine-in" conformation has been suggested to be a characteristic of the active state of MHCK-A. In MHCK-A, N781 and L782 have been suggested to determine the accessibility of the active site. Given their similar spatial location and orientations, a similar role for the analogous $\mathrm{N} 299$ and L300 in eEF-2K $\mathrm{K}_{\mathrm{TR}}$ can be envisaged. The conserved D766 in MHCK-A was phosphorylated in some of the structures. This phospho-aspartyl species has been proposed to represent an intermediate on the pathway to substrate phosphorylation. We do not find evidence of phosphorylation on the equivalent residue (D284) in eEF-2 $\mathrm{K}_{\mathrm{TR}}$ in the $\mathrm{CaM} \bullet p \mathrm{eEF}-2 \mathrm{~K}_{\mathrm{TR}}$ complex. None of the MHCK-A structures used in the overlay contain $p \mathrm{D} 766$. $\mathbf{b}$, Overlay of the KD of $p$ eEF- $2 \mathrm{~K}_{\mathrm{TR}}$ in the $\mathrm{CaM} \cdot p \mathrm{eEF}-2 \mathrm{~K}_{\mathrm{TR}}$ complex (green) and MHCK-A KDs in their nucleotide-bound (yellow) or nucleotide-free structures (pink) with the corresponding Pand the N/D-loops colored orange and magenta, respectively. A closed conformation of the P-loop relative to the N/D-loop in the presence of nucleotide is evident. This closed configuration is also

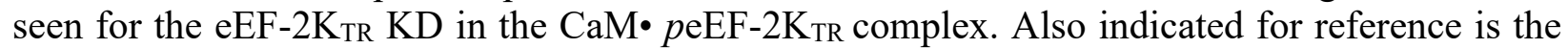
longer $\beta 6-\beta 7-$ loop of eEF- $2 \mathrm{~K}_{\mathrm{TR}}$ that contacts $\mathrm{CaM}_{\mathrm{N}}$ in the $\mathrm{CaM} \bullet p$ eEF- $2 \mathrm{~K}_{\mathrm{TR}}$ complex. The following MHCK-A structures were used in the analysis: for a, 5E9E (bound to AMP-PNP), 3LKM (bound to AMP), 3LMI (D766A mutant bound to ATP), 3LLA (bound to AMPPCP), and 4ZS4 (D756A mutant bound to ATP); for b, 3LLA, 3LMH ( $p$ D766, bound to ADP), 4ZS4, 3PDT 
(C-terminally truncated bound to ADP), 3LKM, 5E9E, 5DYJ (D663A mutant bound to AMP), 4ZME ( $p$ D766, bound to adenosine), 4ZMF ( $p$ D766, bound to AMP), and 5E4H (apo) in b. Note that the only open conformation of the P-loop corresponds to the apo-enzyme $(5 \mathrm{E} 4 \mathrm{H})$. In these structures, D663 was found to be phosphorylated. We do not find evidence of phosphorylation on the equivalent D184 in the structure of the CaM•peEF-2K $\mathrm{K}_{\mathrm{TR}}$ complex. Structural alignments were obtained using the DALI server. 


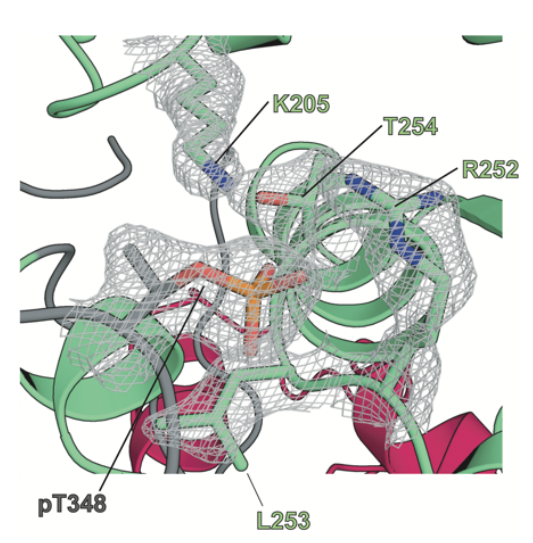

Extended Data Fig. 4 T348 is phosphorylated in the CaM•peEF-2KTR complex. 2Fo-Fc map contoured at $1.5 \sigma$, illustrating $p$ T348 engaged at the PBP. The side-chains of key residues K205, R252, L253, and T254 are shown. 
a

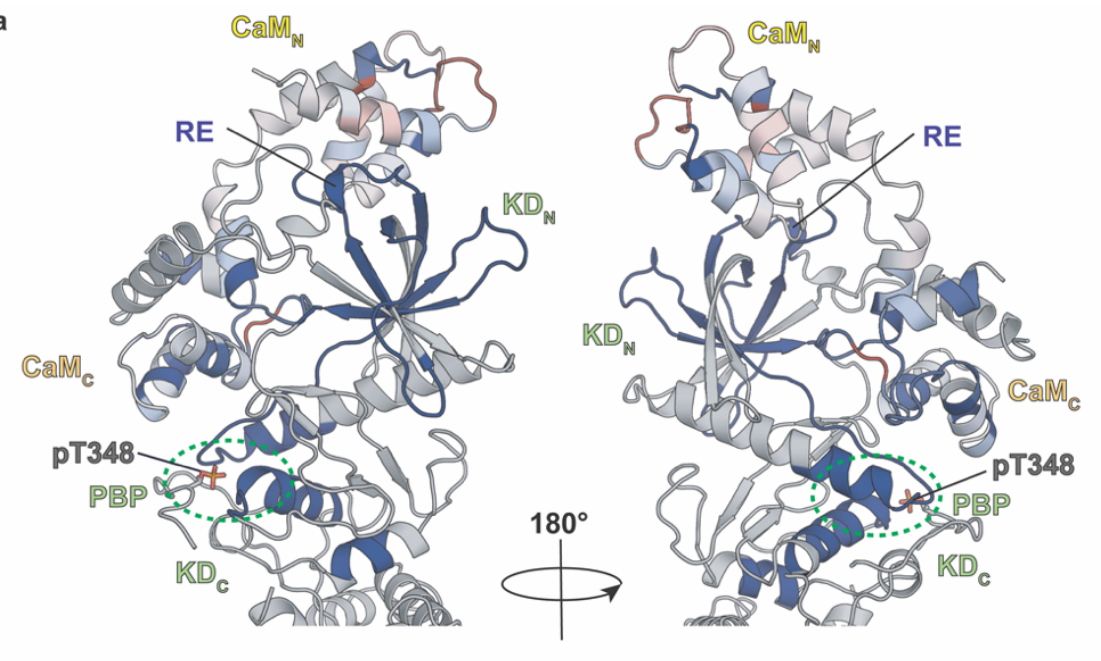

b

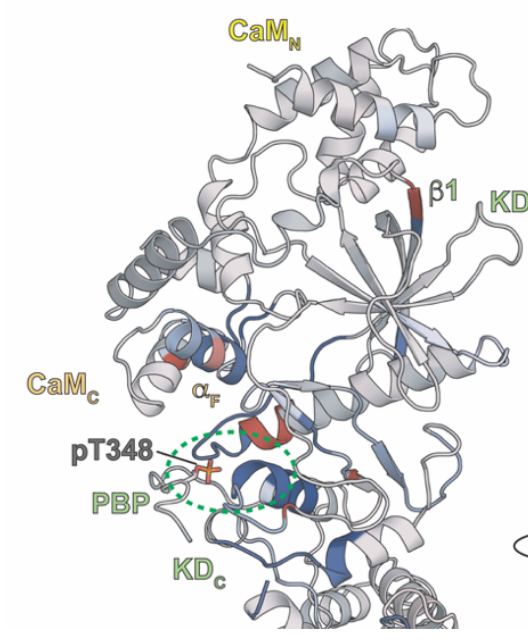

Deprotection/Protection

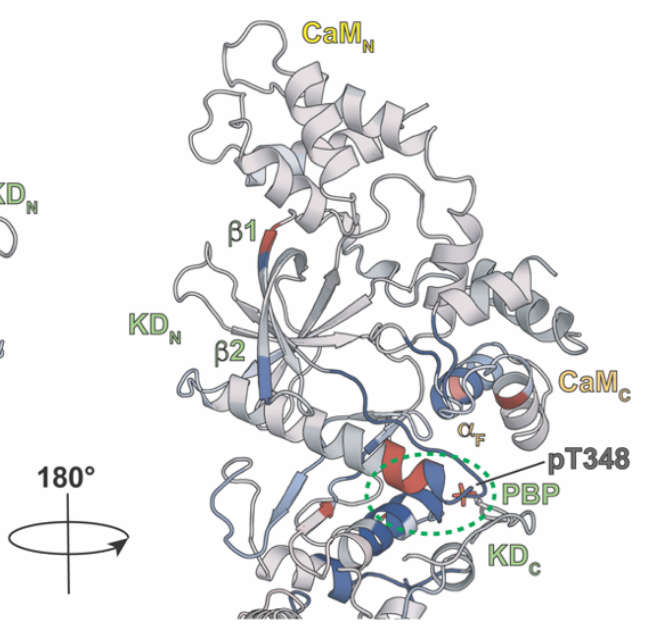

Extended Data Fig. 5 HXMS measurements reveal changes in solvent protection upon complex formation and T348 phosphorylation. a, Changes in solvent protection for the eEF$2 \mathrm{~K}_{\mathrm{TR}} \mathrm{KD}$ and $\mathrm{CaM}$ upon formation of the CaM•eEF- $2 \mathrm{~K}_{\mathrm{TR}}$ complex (unphosphorylated $\mathrm{T} 348$ ) from HXMS measurements, described previously, are plotted on the structure of the CaM•peEF-2 $\mathrm{K}_{\mathrm{TR}}$ complex. Shades of blue or red indicate statistically significant increases or decreases in protection, respectively, upon complex formation. Increased protection is seen throughout $\mathrm{CaM}_{\mathrm{C}}$, on $\mathrm{KD}_{\mathrm{N}}$, including on the $\mathrm{RE}$, and on $\mathrm{KD}_{\mathrm{C}}$ near the $\mathrm{PBP}$ (indicated by the green dashed oval). This suggests the intimate coupling of the structural dynamics of $\mathrm{CaM}_{\mathrm{C}}$ to key regulatory elements within the KD. Changes for $\mathrm{CaM}_{\mathrm{N}}$ are heterogeneous and more modest. $\mathbf{b}$, Changes in solvent protection upon phosphorylation on T348 comparing the CaM•eEF- $2 \mathrm{~K}_{\mathrm{TR}}$ and $\mathrm{CaM} \bullet p \mathrm{eEF}-2 \mathrm{~K}_{\mathrm{TR}}$ complexes (shades of blue indicate increased protection upon phosphorylation on T348). Changes, in this case, are more local and seen at the PBP and on the proximal $\mathrm{CaM}_{\mathrm{C}}$, especially on $\alpha \mathrm{F}$. Some isolated changes are also seen on $\mathrm{KD}_{\mathrm{N}}(\beta 1$ and $\beta 2)$. 


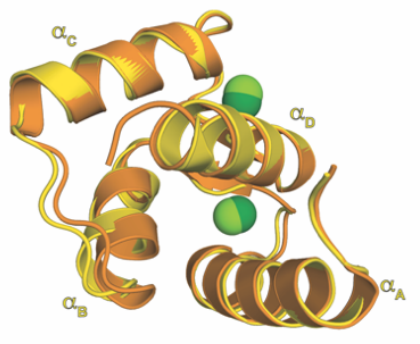

c

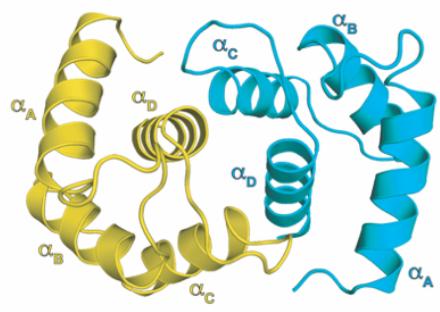

b
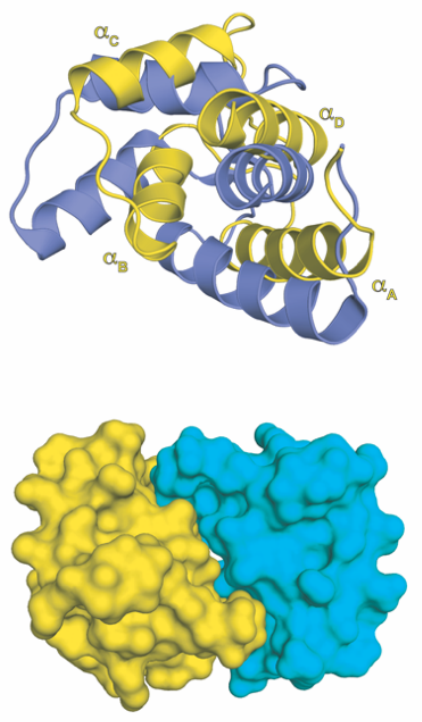

Time (s)

d

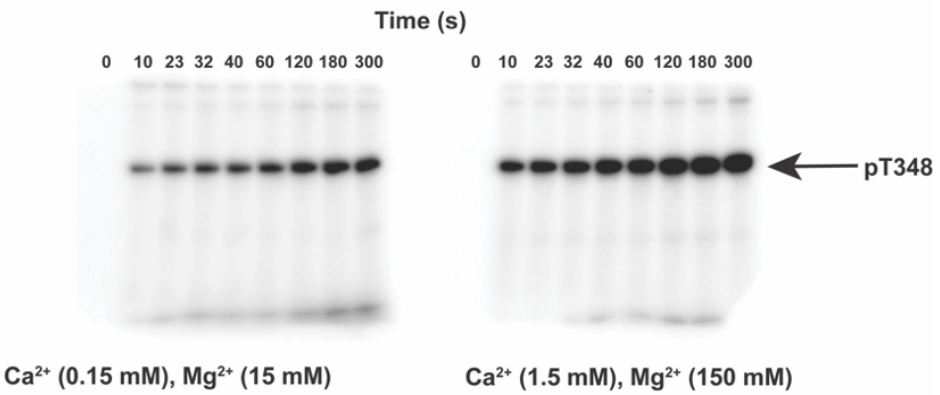

Extended Data Fig. $6 \mathrm{CaM}_{\mathrm{N}}$ adopts a closed conformation in the CaM•peEF-2K $\mathrm{K}_{\mathrm{TR}}$ complex. a, Conformation of $\mathrm{CaM}_{\mathrm{N}}$ in the CaM•peEF-2K $\mathrm{K}_{\mathrm{TR}}$ complex (yellow) or that bound to $\mathrm{Mg}^{2+}$ (orange; PDB: $3 \mathrm{UCW}$ ); the corresponding bound divalent cations are shown as light- or dark-green spheres. b, A comparison of the conformation of $\mathrm{CaM}_{\mathrm{N}}$ in the CaM•peEF- $2 \mathrm{~K}_{\mathrm{TR}}$ complex (yellow) with that in the NMR structure of the complex with CTM-pep in the presence of $\mathrm{Ca}^{2+}$. $\mathrm{CaM}_{\mathrm{N}}$ engages peEF$2 \mathrm{~K}_{\mathrm{TR}}$ in an $\mathrm{Mg}^{2+}$-bound "closed" conformation rather than a $\mathrm{Ca}^{2+}$-bound "open" conformation as in the CTM-pep complex. The metal ions have been omitted for clarity. c, Interactions between two neighboring $\mathrm{CaM}_{\mathrm{N}}$ units related by symmetry within the crystal are shown as ribbons (left) or surfaces (right). The extensive crystal contacts involving $\alpha_{C}$ and $\alpha_{D}$ (the interactions with peEF$2 \mathrm{~K}_{\mathrm{TR}}$ largely involve hydrophobic residues on $\alpha_{\mathrm{A}}$ and $\alpha_{\mathrm{B}}$ ) suggest that this arrangement stabilizes the complex within the lattice. $\mathbf{d}, \mathrm{eEF}-2 \mathrm{~K}_{\mathrm{TR}}$ retains the ability to efficiently auto-phosphorylate on T348 under conditions where the $\mathrm{Ca}^{2+}$ to $\mathrm{Mg}^{2+}$ ratio (1:100) is similar to that used for crystallization. The time-courses for T348 auto-phosphorylation measured using two different sets of concentrations for $\mathrm{Ca}^{2+}$ and $\mathrm{Mg}^{2+}$ while holding their ratio constant are shown. 
a

b
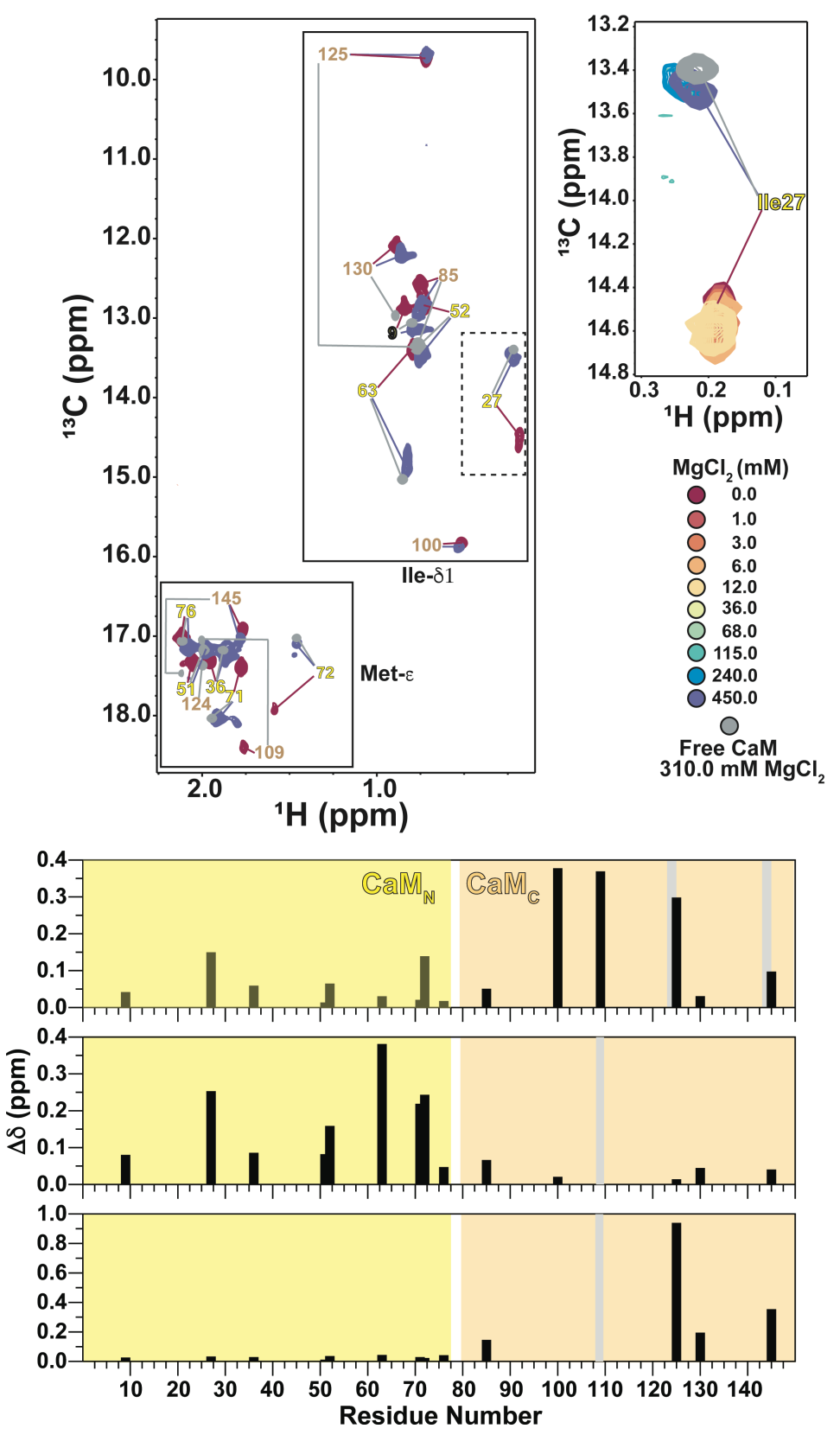

Extended Data Fig. $7 \mathrm{Mg}^{2+}$ partitions exclusively to $\mathrm{CaM}_{\mathrm{N}}$ in the CaM•peEF-2K $\mathrm{KR}_{\mathrm{TR}}$ complex a, Overlays of ${ }^{13} \mathrm{C},{ }^{1} \mathrm{H}$ HMQC spectra $(800 \mathrm{MHz})$ of IM-labeled CaM in the CaM•peEF-2K $\mathrm{K}_{\mathrm{TR}}$ complex in a buffer containing $\mathrm{CaCl}_{2}(300 \mu \mathrm{M})$ with varying concentrations of $\mathrm{MgCl}_{2}$. The resonances of $\mathrm{CaM}$ alone in the presence of a saturating amount of $\mathrm{MgCl}_{2}$ are shown in grey for reference. The residues belonging to $\mathrm{CaM}_{\mathrm{N}}$ or $\mathrm{CaM}_{\mathrm{C}}$ are labeled in yellow and brown font, respectively. Only the initial (red, $0 \mathrm{mM} \mathrm{MgCl}_{2}$ ) and final (navy-blue, $450 \mathrm{mM} \mathrm{MgCl} 2$ ) points of the $\mathrm{Mg}^{2+}$ titration are shown on the left panel. The entire titration course for Ile27, a representative $\mathrm{CaM}_{\mathrm{N}}$ resonance, is shown on the right panel. It is evident from the spectra that the $\mathrm{CaM}_{\mathrm{N}}$ resonances converge to those of the $\mathrm{Mg}^{2+}$-bound, $p$ eEF- $2 \mathrm{~K}_{\mathrm{TR}}$-free state at very high concentrations of $\mathrm{Mg}^{2+}$. The perturbations experienced by the $\mathrm{CaM}_{\mathrm{C}}$ resonances are significantly smaller, and they remain very close to their original positions even at $450 \mathrm{mM} \mathrm{MgCl}$. For $\mathrm{CaM}_{\mathrm{C}}$, the only 
apparent effect is seen for Met109 (at the CTM-binding pocket), which is progressively broadened and fully disappears at the highest $\mathrm{MgCl}_{2}$ concentration. Multiple exchange regimes are seen for the $\mathrm{CaM}_{\mathrm{N}}$ resonances. Resonances whose initial and final positions in the $\mathrm{Mg}^{2+}$-titration are separated by more than $\sim 100 \mathrm{~Hz}$ (e.g., Ile27, Met72) are in a slow exchange regime, suggesting that $\mathrm{Ca}^{2+} / \mathrm{Mg}^{2+}$ exchange occurs on the ms timescale. $\mathbf{b}$, Chemical shift perturbations induced by peEF-2K $\mathrm{K}_{\mathrm{TR}}$ on IM-labeled CaM in the presence of $\mathrm{Ca}^{2+}$ (and the absence of $\mathrm{Mg}^{2+}$ ) are shown on the top panel. Fully broadened resonances are indicated by the grey bars. The largest perturbations are observed for $\mathrm{CaM}_{\mathrm{C}}$. The patterns of perturbations are similar to that reported by the presence of eEF-2K $\mathrm{K}_{\mathrm{TR}}$ (unphosphorylated T348), suggesting that the CaM-recognition modes of eEF-2KTR and $p$ EEF-2KTR are similar in the two cases. Perturbations induced by $\mathrm{Mg}^{2+}\left(450 \mathrm{mM} \mathrm{MgCl}_{2}\right)$ on IM-labeled $\mathrm{CaM}$ in the CaM•peEF-2K TR complex (containing $300 \mu \mathrm{M} \mathrm{CaCl}_{2}$ ) are shown on the middle panel. Significant perturbations are seen on $\mathrm{CaM}_{\mathrm{N}}$; minimal perturbations are seen for $\mathrm{CaM}_{\mathrm{C}}$ (except for the Met109 resonance that is broadened to below the noise). Perturbations in IM-labeled CaM comparing the CaM•peEF-2 $\mathrm{K}_{\mathrm{TR}}$ complex in buffer containing $300 \mu \mathrm{M} \mathrm{CaCl}_{2}$ and $450 \mathrm{mM} \mathrm{MgCl} 2$, with CaM alone in the presence of $310 \mathrm{mM} \mathrm{MgCl}_{2}$, are shown on the bottom panel. The data suggest that, at high concentrations, $\mathrm{Mg}^{2+}$ replaces $\mathrm{Ca}^{2+}$ on $\mathrm{CaM}_{\mathrm{N}}$, leading to its disengagement from $p e E F-2 \mathrm{~K}_{\mathrm{TR}}$. 
a
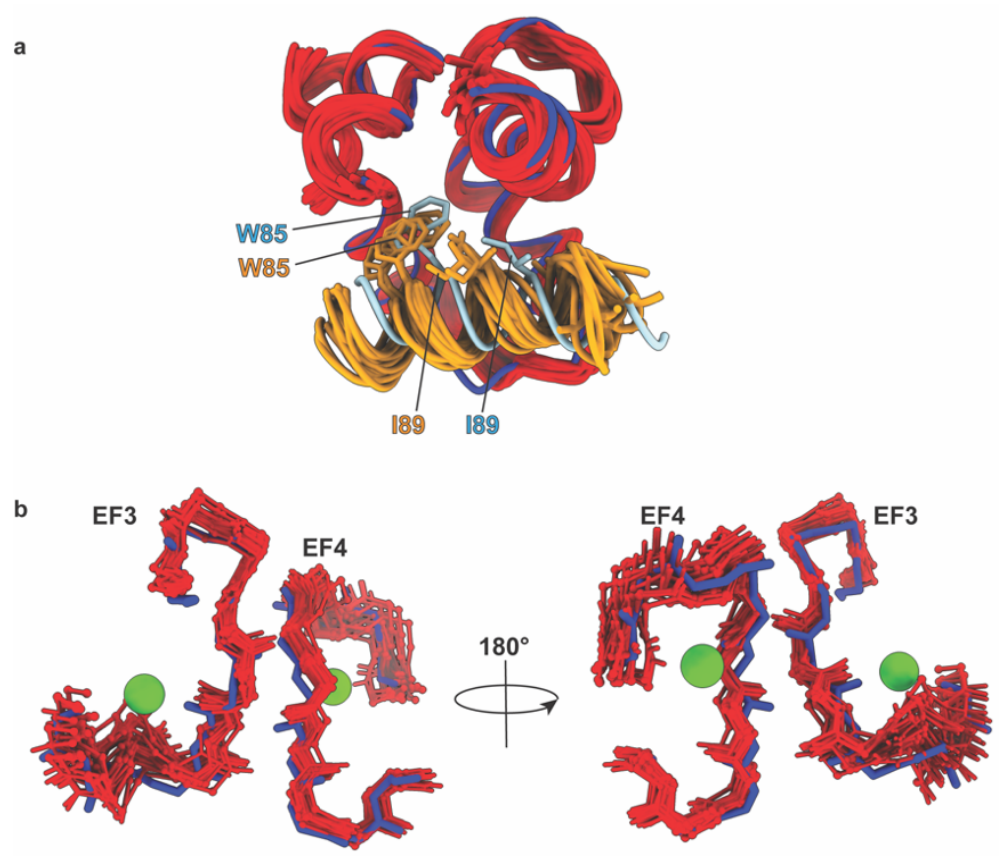

Extended Data Fig. 8 The CaM $/$ CTM module adopts slightly different conformations in the peEF-2K $K_{\text {TR }}$ and CTM-pep complexes. a, Comparison of the CaMC $/ \mathrm{CTM}$ modules in the NMR ensemble of the complex of CaM with CTM-pep (PDB: $5 \mathrm{~J} 8 \mathrm{H}$ ) or in the CaM•peEF-2K $\mathrm{K}_{\mathrm{TR}}$ complex. $\mathrm{CaM}_{\mathrm{C}}(\mathrm{CTM})$ is shown in red (orange) and dark-blue (light-blue) in the NMR and crystal structures, respectively. The CTM is displaced by approximately half a helical turn within $\mathrm{CaM}_{\mathrm{C}}$ in the crystal structure compared to the NMR ensemble. The W85 and I89 side-chains are shown for only 5 representative structures of the NMR ensemble for ease in visualization. The W85 sidechain occupies a similar spatial position, on average, in the crystal structure and the NMR ensemble. The orientation of the side-chains of I89 and A92 (hidden) are significantly different in the two cases. $\mathbf{b}$, Backbone traces of the $\mathrm{Ca}^{2+}$ binding EF-hands (EF3, EF4) of CaM $\mathrm{C}_{\mathrm{C}}$ in the NMR ensemble (red) of the CaM•CTM-pep complex illustrate their displacement from the spatial position seen in the crystal structure of the CaM•peEF- $2 \mathrm{~K}_{\mathrm{TR}}$ complex (blue). These displacements make the $\mathrm{CaM}_{\mathrm{C}}$ EF-hands unsuitable for coordinating $\mathrm{Ca}^{2+}$ in the $\mathrm{CaM} \cdot \mathrm{CTM}$-pep complex. 


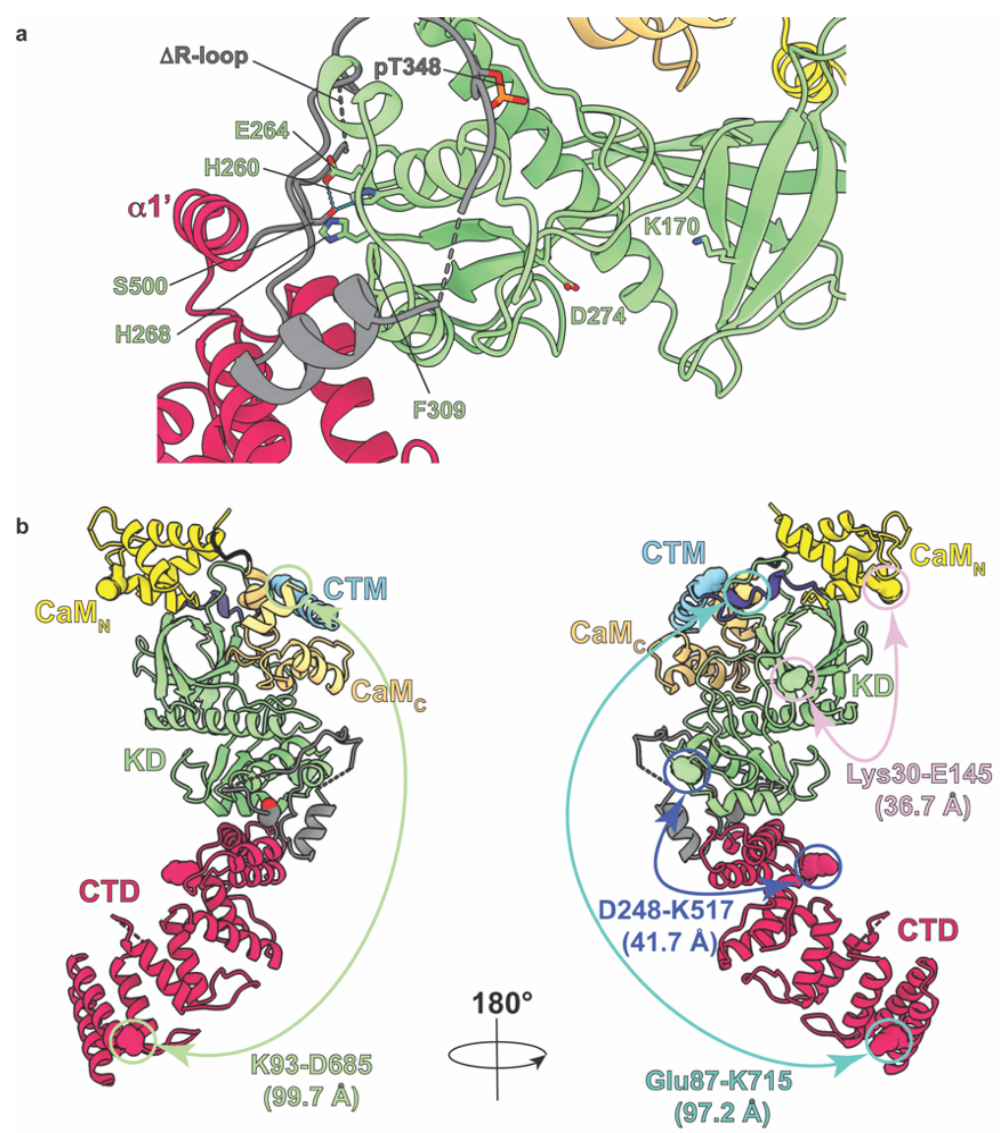

Extended Data Fig. 9 Auto-phosphorylation on S500 requires conformational changes. a, The secondary site of activating auto-phosphorylation (S500) is buried in a pocket within $\mathrm{KD}_{\mathrm{C}}$, being stabilized through hydrogen bonds involving the side-chains of the $\mathrm{Zn}^{2+}$-coordinating $\mathrm{H} 260$, and E264, at a significant distance from the catalytic site (e.g., the S500,O $\gamma-\mathrm{D} 274, \mathrm{O} \delta 1$ and the $\mathrm{S} 500, \mathrm{O} \gamma-\mathrm{K} 170, \mathrm{~N} \zeta$ distances are 20.0 and $29.7 \AA$, respectively; D274 is the putative catalytic base) suggesting that auto-phosphorylation at this position in cis would require significant conformational rearrangements including a partial unfolding of $\alpha 1^{\prime}$. Also shown for reference is the position of $p$ T348 bound at the PBP. b, Unambiguous intermolecular (between CaM and eEF$\left.2 \mathrm{~K}_{\mathrm{TR}}\right)$ and intramolecular $(\mathrm{CTM} / \mathrm{CTD}, \mathrm{KD} / \mathrm{CTD})$ crosslinks assigned for the CaM•eEF-2K $\mathrm{K}_{\mathrm{TR}}$ complex using the "zero-length" carbodiimide crosslinker EDC. While the two shorter C $\alpha-C \alpha$ distances could be accommodated within a 1:1 complex through conformational flexibility, the longer distances would require substantial structural distortions to enable close contact of the KD with the CTD, and of the CTD with CaM, as proposed in an earlier computational model. However, all these crosslinks would be compatible with a 2:2 complex with an antiparallel assembly of the constituent CaM•eEF-2K $\mathrm{K}_{\text {TR }}$ units. Such an arrangement would also facilitate in trans S500 autophosphorylation through smaller conformational changes. CaM and eEF- $2 \mathrm{~K}_{\mathrm{TR}}$ residues are indicated by three- and one-letter codes, respectively. 


\section{Extended Data Table S1. Data collection and refinement statistics}

\begin{tabular}{|c|c|}
\hline Data Collection & \\
\hline Beamline & NSLS-II BEAMLINE 19-ID \\
\hline Wavelength $(\AA)$ & 0.97946 \\
\hline Space Group & $\mathrm{P} 3{ }_{1} 21$ \\
\hline Cell dimensions: & \\
\hline$a, b, c(\AA)$ & 58.5058 .50365 .78 \\
\hline$\alpha, \beta, \gamma\left({ }^{\circ}\right)$ & 9090120 \\
\hline Resolution $(\AA)$ & $\begin{array}{c}121.92 .34 \\
(2.382,2.342)\end{array}$ \\
\hline $\mathrm{R}_{\text {merge }}$ & $0.362(1.778)$ \\
\hline$I / \sigma(I)$ & $14.5(2.5)$ \\
\hline Completeness $(\%)$ & $100(100)$ \\
\hline Redundancy & $25.9(24.5)$ \\
\hline Wilson B-factor & 14.99 \\
\hline Number of unique reflections & $32010(1494)$ \\
\hline Refinement & \\
\hline Resolution range $(\AA)$ & $50.66-2.34$ \\
\hline No. reflections & 31982 \\
\hline $\mathrm{R}_{\text {work }} / \mathrm{R}_{\text {free }}$ & $0.2293 / 0.2520$ \\
\hline Number of non-hydrogen atoms & \\
\hline Macromolecules & 5117 \\
\hline Ligands & 8 \\
\hline Solvent & 144 \\
\hline Protein residues & 637 \\
\hline RMS (bond) $(\AA)$ & 0.003 \\
\hline RMS (angles) $\left(^{\circ}\right.$ ) & 0.533 \\
\hline Ramachandran Plot & \\
\hline Most favored (\%) & 91.1 \\
\hline Additionally favored (\%) & 8.7 \\
\hline Generously favored (\%) & 0.2 \\
\hline Disallowed $(\%)$ & 0.0 \\
\hline Rotamer outliers (\%) & 0.38 \\
\hline Clash-score & 4.29 \\
\hline Average B-factor $\left(\AA^{2}\right)$ & 35.37 \\
\hline Macromolecules & 35.6 \\
\hline Ligands & 29.35 \\
\hline Solvent & 27.61 \\
\hline PDB accession code & $7 \mathrm{SHQ}$ \\
\hline
\end{tabular}

Values in parentheses are for the highest resolution shell. $\mathrm{R}_{\text {merge }}=\sum|(I-<I>)| / \sum I$, where $I$ is the observed intensity. 